\title{
Traumatic Brain Injury
}

IN THE UNITED STATES

Emergency Department Visits, Hospitalizations and Deaths 2002-2006 


\title{
Traumatic Brain Injury
}

\section{IN THE UNITED STATES}

Emergency Department Visits, Hospitalizations and Deaths 2002-2006

\author{
U.S. DEPARTMENT OF HEALTH AND HUMAN SERVICES \\ Centers for Disease Control and Prevention \\ National Center for Injury Prevention and Control
}

www.cdc.gov/TraumaticBrainInjury 


\section{AUTHORS}

\section{Mark Faul, PhD, MS}

National Center for Injury Prevention and Control

Division of Injury Response

\section{Likang Xu, MD, MS}

National Center for Injury Prevention and Control

Division of Injury Response

\section{Marlena M. Wald, MPH, MLS}

National Center for Injury Prevention and Control

Division of Injury Response

\section{Victor G. Coronado, MD, MPH}

National Center for Injury Prevention and Control

Division of Injury Response

\section{ACKNOWLEDGMENTS}

The authors would like to thank Vikas Kapil and Lisa McGuire for their editorial comments, Karen Thomas for her programming assistance and Michael Lionbarger. The authors also offer sincere appreciation to the many advisors to this report, including Kevin Webb and Dionne Williams, for their guidance.
Traumatic Brain Injury in the United States: Emergency Department Visits, Hospitalizations and Deaths 2002-2006 is a publication of the National Center for Injury Prevention and Control, Centers for Disease Control and Prevention.

Centers for Disease Control and Prevention Thomas R. Frieden, MD, MPH, Director

\section{National Center for Injury Prevention and Control} Robin Ikeda, MD, MPA, Acting Director

Division of Injury Response Richard C. Hunt, MD, FACEP, Director

The findings and conclusions in this report are those of the authors and do not necessarily represent the official position of the Centers for Disease Control and Prevention (CDC).

SUGGESTED CITATION: Faul M, Xu L, Wald MM, Coronado VG. Traumatic Brain Injury in the United States: Emergency Department Visits, Hospitalizations and Deaths 2002-2006. Atlanta (GA): Centers for Disease Control and Prevention, National Center for Injury Prevention and Control; 2010. 
THE POWER OF DATA

A Message from a TBI Survivor

"Society is more likely to take action against the ravages of traumatic brain injury if it understands how pernicious, pervasive, and huge the problem is.

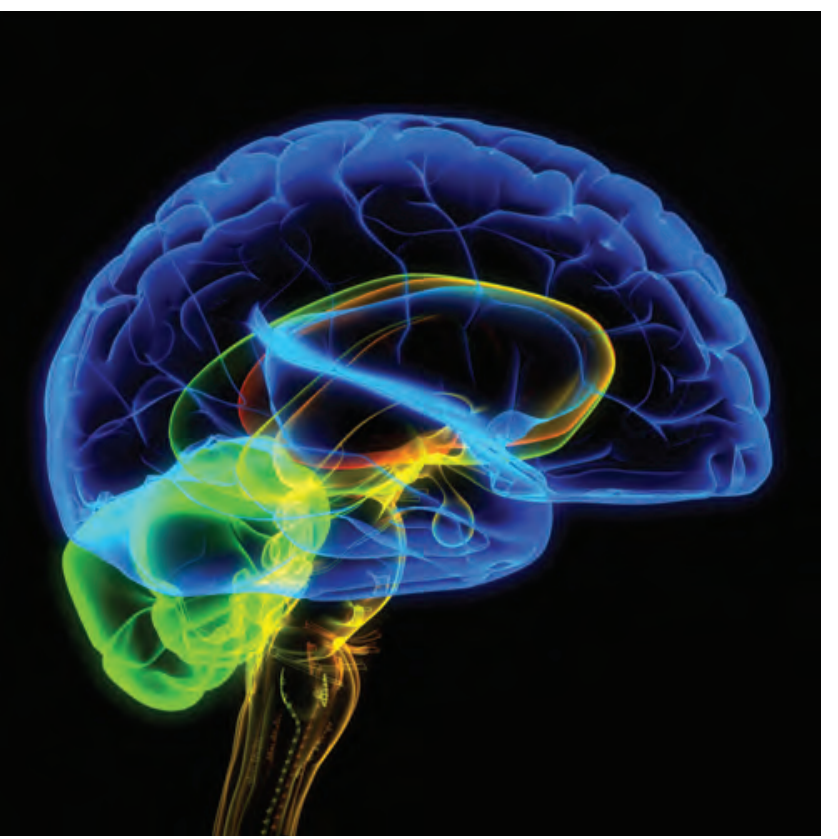

This body of work is a vital tool for those who devise the strategies for prevention and treatment. However, a critical dimension will be lost if one sees it only as data, if one does not try to put even a fleeting face behind the numbers. They represent people who - if they survived - have had their lives significantly affected. Through research, we are finding better ways to prevent injury and improve acute care. We who are injured may experience improvement both in function and the quality of our lives when we have access to rehabilitation and support to develop and utilize our remaining strengths and abilities. With so many lives affected, we seek and have the potential for independence, to have the chance to move beyond our disabilities and give back to society.

As a survivor, as a disabled physician, I applaud this publication as a step toward making that possible." 


\section{TABLE OF CONTENTS}

\begin{tabular}{ll} 
Executive Summary & $\mathbf{5}$ \\
\hline Key Findings & 7 \\
Background & 8 \\
Report Contents and Organization & 9 \\
Overview & 11 \\
\hline Annual Number of TBIs & 13 \\
TBI as a Proportion of All Injuries & 14 \\
TBI by Age Group & 15 \\
TBI by Sex & 16 \\
TBI by External Cause & 17 \\
Summary of Findings External TBI Causes & 21 \\
Conclusion & 21
\end{tabular}

\section{Appendix A: Tables}

23

Total TBI-Related Emergency Department Visits,

Hospitalizations, and Deaths

TBI-Related Emergency Department Visits

by Age Group and Disposition
TBI-Related Emergency Department Visits by Age Group and Sex

TBI-Related Emergency Department Visits by Age Group and Race

TBI-Related Emergency Department Visits by Age Group and External Cause

TBI-Related Emergency Department Visits by Age Group and Motor - Vehicle Cause

TBI-Related Emergency Department Visits by Age Group and Payment Source

TBI-Related Hospitalizations

by Age Group and Disposition

TBI-Related Hospitalizations by Age Group and Sex

TBI-Related Hospitalizations by Age Group and Race

TBI-Related Hospitalizations by Age Group and External Cause

TBI-Related Hospitalizations by Age Group and Motor - Vehicle Cause

TBI-Related Hospitalizations by Age Group and Payment Source 
Appendix A: Tables continued

TBI-Related Deaths by Age Group and Sex

TBI-Related Deaths by Age Group and Race

TBI-Related Deaths by Age Group and External Cause

TBI-Related Deaths by Age Group

and Motor - Vehicle Cause

Annual Estimates of All TBI from 2002-2006

Annual Rate Estimates of Fall-Related TBI

Among Children Aged 0 - 14 Years

Annual Rate Estimates of Fall-Related TBI

Among Adults Aged 65 Years and Older

Appendix B: Methods and Data Sources

38

39

40

41

42

43

43

\section{Data Sources}

Identification of TBI Cases

External Cause of Injury

Population Data

Statistical Analysis

Limitations

Index of Tables and Figures

References
63

45

51

55 


\section{EXECUTIVE SUMMARY}

Traumatic brain injury (TBI) is an important public health problem in the United States. TBI is frequently referred to as the "silent epidemic" because the complications from TBI, such as changes affecting thinking, sensation, language, or emotions, may not be readily apparent. In addition, awareness about TBI among the general public is limited.

Through the TBI Act of 1996 (Public Law 104-166), Congress first charged the Centers for Disease Control and Prevention (CDC) with "determining the incidence and prevalence of traumatic brain injury in all age groups in the general population of the United States." In response, CDC has produced, Traumatic Brain Injury in the United States: Emergency Department Visits, Hospitalizations and Deaths 2002-2006.
Population-based data on TBI are critical to understanding the impact of TBI on the American people. This report presents data on TBI-related emergency department visits, hospitalizations, and deaths for the years 2002 through 2006 and can be used to determine the number of TBIs occurring each year, groups most affected, and the leading causes of TBI. This important information can be used to document the need for TBI prevention, to identify research and education priorities, and to support the need for services among individuals living with a TBI.

This report is an update to CDC's previously published report released in 2004 and is intended as a reference for policymakers, health care and service providers, educators, researchers, advocates, and others interested in knowing more about the impact of TBI in the United States. ${ }^{1}$ 


\section{KEY FINDINGS IN THIS REPORT}

\section{TBI in the United States}

- An estimated 1.7 million people sustain a TBI annually. Of them:

- 52,000 die,

-275,000 are hospitalized, and

- 1.365 million, nearly $80 \%$, are treated and released from an emergency department.

- TBI is a contributing factor to a third (30.5\%) of all injury-related deaths in the United States.

TBI by Age

- Children aged o to 4 years, older adolescents aged 15 to 19 years, and adults aged 65 years and older are most likely to sustain a TBI.

- Almost half a million $(473,947)$ emergency department visits for TBI are made annually by children aged 0 to 14 years.

- Adults aged 75 years and older have the highest rates of TBI-related hospitalization and death.

TBI by Sex

- In every age group, TBI rates are higher for males than for females.

- Males aged 0 to 4 years have the highest rates for TBI-related emergency department visits, hospitalizations, and deaths combined.

\section{TBI by External Cause}

- Falls are the leading cause of TBI. Rates are highest for children aged 0 to 4 years and for adults aged 75 years and older.

- Falls result in the greatest number of TBI-related emergency department visits $(523,043)$ and hospitalizations $(62,334)$.

- Motor vehicle-traffic injury is the leading cause of TBI-related death. Rates are highest for adults aged 20 to 24 years.

\section{Additional TBI Findings*}

- There was an increase in TBI-related emergency department visits (14.4\%) and hospitalizations (19.5\%) from 2002 to 2006

- There was a $62 \%$ increase in fall-related TBI seen in emergency departments among children aged 14 years and younger from 2002 to 2006.

- There was an increase in fall-related TBIs among adults aged 65 and older; $46 \%$ increase in emergency department visits, $34 \%$ increase in hospitalizations, and $27 \%$ increase in TBI-related deaths from 2002 to 2006

* Estimates based on one year of data can produce varied results. 


\section{BACKGROUND}

Each year, traumatic brain injuries (TBI) contribute to substantial number of deaths and cases of permanent disability. A TBI is caused by a bump, blow or jolt to the head or a penetrating head injury that disrupts the normal function of the brain. The severity of a TBI may range from "mild" (a brief change in mental status or consciousness) to "severe" (an extended period of unconsciousness or amnesia after the injury).

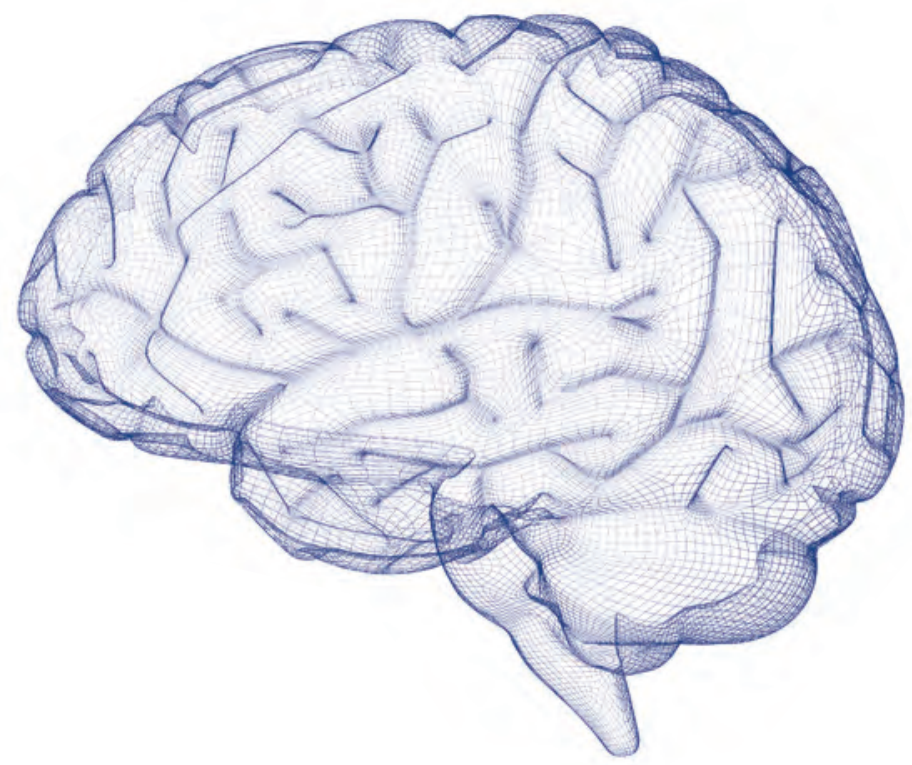




\section{REPORT CONTENTS AND ORGANIZATION}

This report presents data about TBI-related emergency department visits, hospitalizations, and deaths in the United States for the years 2002 through 2006. The findings are organized into two main sections of Overview and Appendices. The Overview summarizes and interprets key findings and the Appendices present detailed data tables and a description of the methods and limitations.

Average annual numbers of TBIs per year and annual rates are both reported. While the annual numbers show the magnitude of the problem, the rates show how a certain group is affected by TBI by relating the number of TBIs to the size of the population. For example, a relatively small number of TBIs occurring in a small population would result in a higher TBI rate than if the same number of TBIs occurred in a larger population. This report helps to answer a variety of questions, such as: "Do males sustain TBIs more often than females?; Are children more likely to sustain a TBI than adults?; and, Are motor vehicle-traffic injuries a substantial cause of TBI among older adults?”
Data in the report include:

- TBI as a Proportion of All Injuries

- TBI by Age

- TBI by Sex

- TBI by Race

- TBI by External Cause

- Additional TBI Findings

State-level data on TBI are not presented in this report. CDC's National Center for Injury Prevention and Control, Division of Injury Response currently funds 30 states to conduct TBI surveillance through the CORE State Injury Program.

For TBI-related death and hospitalization data by participating states, download a copy of the State Injury Indicators Report: Fourth Edition-2005 Data at no cost or search the Injury Indicators Web-based Query System, both available at www.cdc.gov/Injury.

Finally, neither this report nor the State Injury Indicators Report: Fourth Edition-2005 Data include TBIs from federal, military, or Veterans Affairs (VA) hospitals. 


\section{FOR MORE INFORMATION}

CDC's Division of Injury Response works to reduce injuries and their adverse health effects. For additional information on TBI, including research, programs, and educational initiatives please visit: www.cdc.gov/TraumaticBrainInjury.

For questions about this report, please contact CDC-INFO@cdc.gov or call 1-800-CDC-INFO (1-800-232-4636).

For media inquiries, please contact CDC's Injury Center Press Officer at (770) 488-4902 between 9:00 am and 5:00 pm EST. If there is an after-hours emergency, please call (404) 639-2888 for instructions on contacting the on-call press officer. 


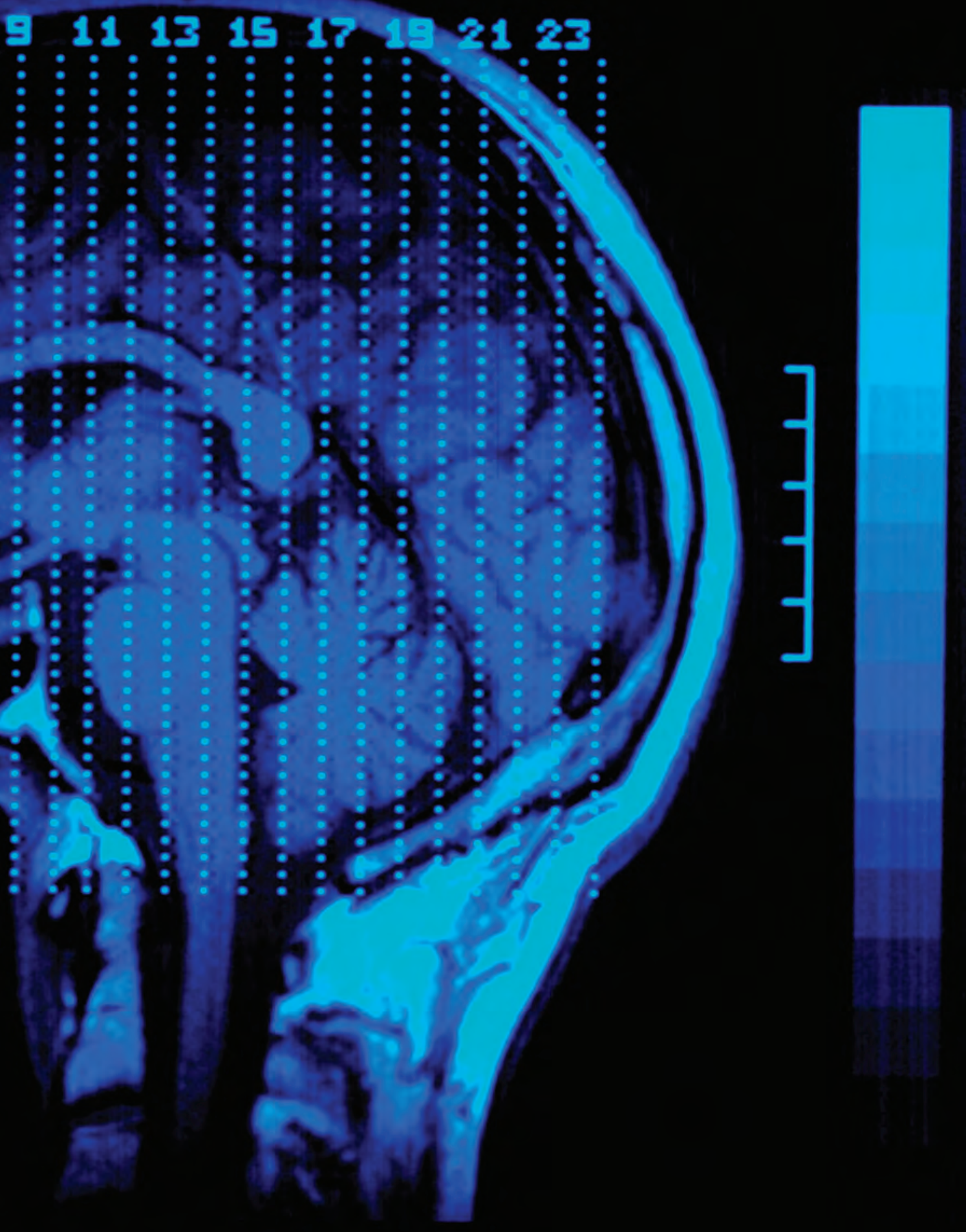

\section{Overview}

Annual Number of TBIs

TBI as a Proportion of All Injuries TBI by Age Group

TBI by Sex

TBI by External Cause

Summary of Findings

External TBI Causes

Conclusion
13

14

15

16

17

21

21 


\section{ANNUAL NUMBER OF TBIS}

FIGURE 1: Estimated Average Annual Number of Traumatic Brain Injury-Related Emergency Department Visits, Hospitalizations, and Deaths, United States, 2002-2006

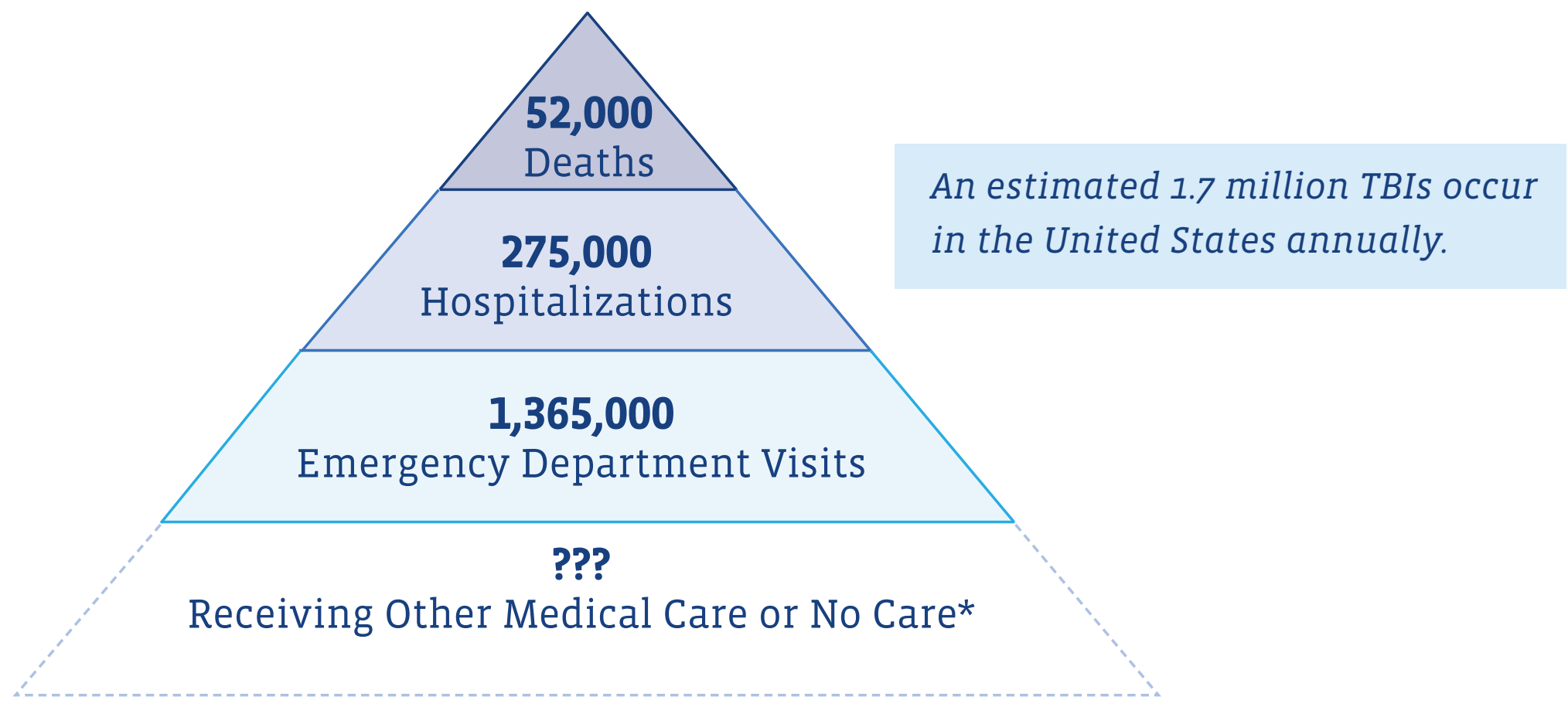

Of the 1.7 million TBIs occurring each year in the United States, 80.7\% were emergency department visits, $16.3 \%$ were hospitalizations, and $3.0 \%$ were deaths.

* Data for this category are not included in this report. See "Limitations" in Appendix B for more information. 


\section{TBI AS A PROPORTION OF ALL INJURIES}

TABLE A: Estimated Percentage of All Injuries and Traumatic Brain Injury-Related Emergency Department Visits, Hospitalizations, and Deaths, United States, 2002-2006

\begin{tabular}{|c|c|c|c|c|c|c|}
\hline & \multirow[b]{2}{*}{ ALL VISITS } & \multicolumn{2}{|c|}{ ALL INJURIES } & \multicolumn{3}{|c|}{ TBIs } \\
\hline & & NUMBER & $\begin{array}{c}\% \text { OF } \\
\text { ALL VISITS }\end{array}$ & NUMBER & $\begin{array}{l}\% \text { OF ALL } \\
\text { INJURIES }\end{array}$ & $\begin{array}{c}\% \text { OF } \\
\text { ALL VISITS }\end{array}$ \\
\hline ED Visits ${ }^{*}$ & $96,839,411$ & $28,697,028$ & 29.6 & $1,364,797$ & 4.8 & 1.4 \\
\hline Hospitalizations\# & $36,693,646$ & $1,826,548$ & 5.0 & 275,146 & 15.1 & 0.7 \\
\hline Deaths & $2,432,714$ & 169,055 & 6.9 & $51,538^{+}$ & 30.5 & 2.1 \\
\hline Total & $135,965,771$ & $30,692,631$ & 22.6 & $1,691,481$ & 5.5 & 1.2 \\
\hline
\end{tabular}

* Persons who were hospitalized, died, or transferred to another facility were excluded.

\# In-hospital deaths and patients who transferred from another hospital were excluded.

+ 128 mortality records (from 2002-2006) were omitted because of missing age information.

The estimated annual average number of emergency department visits, hospitalizations and deaths for all injuries is in Table A. TBIs comprise $4.8 \%$ of all injuries seen in emergency department visits and $15.1 \%$ of all hospitalizations. Of all the injuryrelated deaths in the United States, TBI was a contributing factor $30.5 \%$ of the time. 


\section{TBI BY AGE GROUP}

\section{COMPARING THE NUMBERS}

TABLE B: Estimated Average Annual Numbers of Traumatic Brain Injury-Related Emergency Department Visits, Hospitalizations, and Deaths, by Age Group, United States, 2002-2006

\begin{tabular}{|c|c|c|c|c|}
\hline AGE GROUP & $\begin{array}{c}\text { EMERGENCY } \\
\text { DEPARTMENT } \\
\text { VISITS }\end{array}$ & HOSPITALIZATIONS & DEATHS & TOTAL \\
\hline $\begin{array}{l}\text { Children } \\
\text { (0-14 years) }\end{array}$ & 473,947 & 35,136 & 2,174 & 511,257 \\
\hline $\begin{array}{l}\text { Older Adults } \\
\text { ( } \geq 65 \text { years) }\end{array}$ & 141,998 & 81,499 & 14,347 & 237,844 \\
\hline
\end{tabular}

The estimated average annual number of TBIs that occur among children aged 0 to 14 years is 511,257 . In contrast the number of TBIs in adults aged 65 years and older is 237,844 . TBI-related emergency department visits accounted for a larger proportion in children (92.7\%) than in older adults (59.7\%).

\section{COMPARING THE RATES}

FIGURE 2: Estimated Average Annual Rates of Traumatic Brain Injury-Related Emergency Department Visits, Hospitalizations, and Deaths, by Age Group, United States, 2002-2006

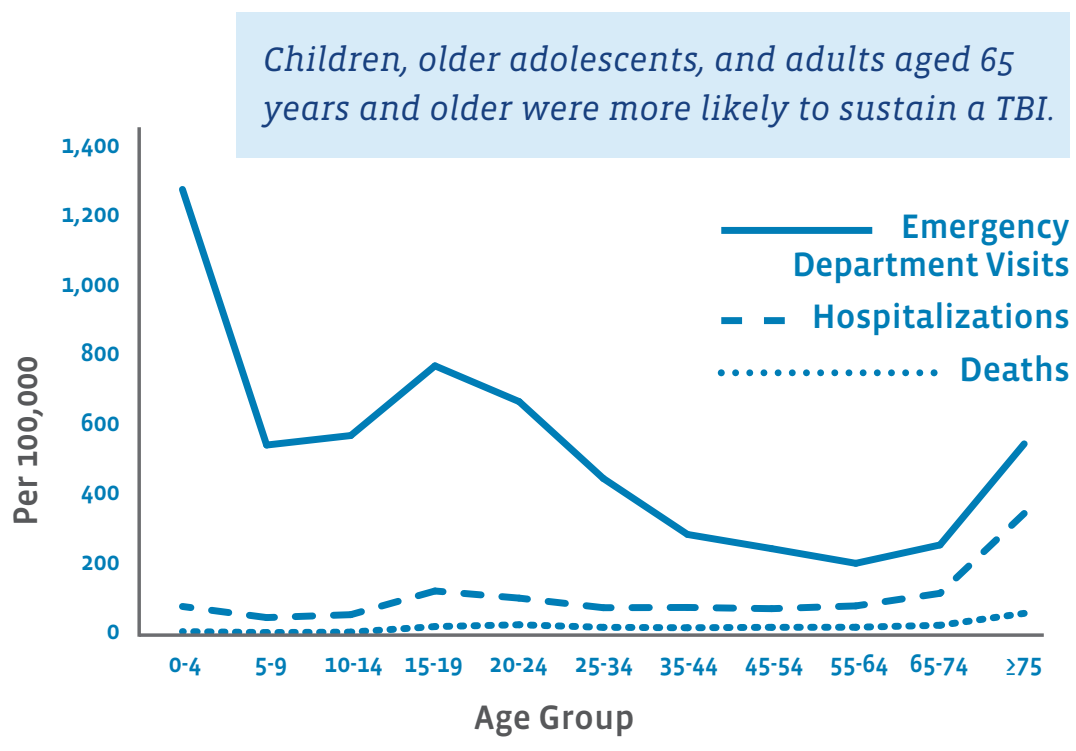

Very young children aged o to 4 years had the highest rate of TBI-related emergency department visits (1,256 per 100,000 population), followed by older adolescents aged 15 to 19 years (757 per 100,000). However, the highest rates of TBI-related hospitalization and death occurred among adults aged 75 years and older (339 per 100,000 and 57 per 100,000, respectively). 


\section{TBI BY SEX}

\section{COMPARING THE NUMBERS}

TABLE C: Estimated Average Annual Numbers of Traumatic Brain Injury-Related Emergency Department Visits, Hospitalizations, and Deaths, by Sex, United States, 2002-2006

\begin{tabular}{|c|c|c|c|c|}
\hline SEX & $\begin{array}{l}\text { EMERGENCY } \\
\text { DEPARTMENT } \\
\text { VISITS }\end{array}$ & HOSPITALIZATIONS & DEATHS & TOTAL \\
\hline Male & 789,925 & 170,257 & 37,994 & 998,176 \\
\hline Female & 574,870 & 104,890 & 13,569 & 693,329 \\
\hline
\end{tabular}

An estimated average annual number of 998,176 TBIs occurred among males compared with 693,329 among females. Overall, approximately 1.4 times as many TBIs occurred among males as among females.

\section{COMPARING THE RATES}

FIGURE 3: Estimated Average Annual Rates of Traumatic Brain Injury-Combined Emergency Department Visits, Hospitalizations, and Deaths, by Sex, United States, 2002-2006

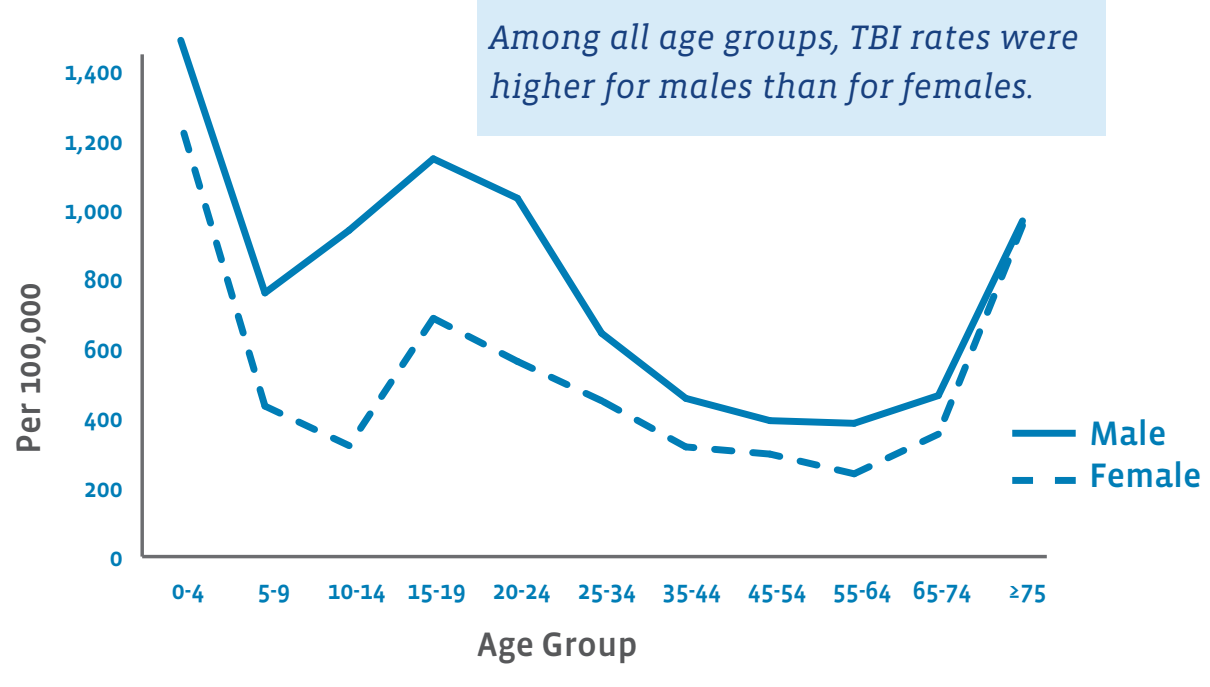

Males aged 0 to 4 years had the highest rates of TBI-related emergency department visits, hospitalizations and deaths combined $(1,451$ per 100,000). Rates were also high for females aged 0 to $4(1,218$ per 100,000), and for both males and females aged 15 to 19 years (896 per 100,000), and 75 years and older (932 per 100,000). 


\section{TBI BY EXTERNAL CAUSE}

\section{COMPARING THE NUMBERS}

TABLE D: Estimated Average Annual Numbers of Traumatic Brain Injury-Related Emergency Department Visits, Hospitalizations, and Deaths, by External Cause, United States, 2002-2006

\begin{tabular}{|c|c|c|c|c|}
\hline CAUSE & $\begin{array}{l}\text { EMERGENC } \\
\text { DEPARTMEN } \\
\text { VISITS }\end{array}$ & HOSPITALIZATIONS & DEATHS & TOTAL \\
\hline Falls & 523,043 & 62,334 & 9,718 & 595,095 \\
\hline $\begin{array}{l}\text { Struck } \\
\text { By/Against }\end{array}$ & 271,713 & 7,791 & 378 & 279,882 \\
\hline $\begin{array}{l}\text { Motor Vehicle- } \\
\text { Traffic }\end{array}$ & 218,936 & 56,864 & 16,402 & 292,202 \\
\hline Assault & 148,471 & 15,341 & 5,813 & 169,625 \\
\hline Other & 108,467 & 27,536 & 19,252 & 155,255 \\
\hline Unknown & 94,165 & 105,282 & 0 & 199,447 \\
\hline
\end{tabular}

An estimated average annual number of 595,095 are fallrelated TBIs, 292,202 are motor vehicle-traffic TBIs, 279,882 are struck by/against events, and 169,625 are assault-related TBIs. Motor vehicle-traffic resulted in the greatest number of TBIrelated deaths; however, falls resulted in the greatest number of emergency department visits and hospitalizations.

\section{COMPARING THE RATES}

FIGURE 4: Estimated Average Annual Rates of Traumatic Brain Injury-Combined Emergency Department Visits, Hospitalizations, and Deaths, by External Cause, United States, 2002-2006

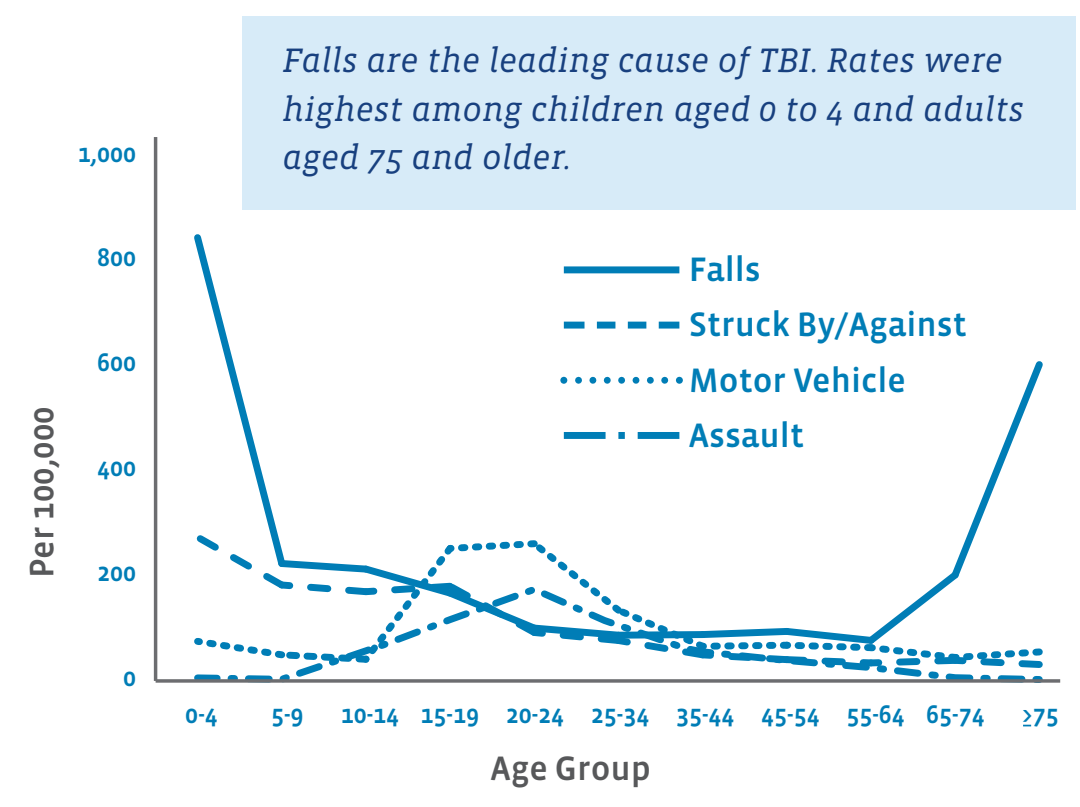

The rate of fall-related TBI was highest among children aged 0 to 4 years (839 per 100,000) and adults aged 75 years and older (599 per 100,000). The rates for both motor vehicle-traffic and assault-related TBI were highest among adults aged 20 to 24 years (261 per 100,000 and 175 per 100,000, respectively). 


\section{TBI BY EXTERNAL CAUSE}

\section{COMPARING THE PERCENTAGES}

FIGURE 5: Estimated Average Percentage of Annual Traumatic Brain Injury-Combined Emergency Department Visits, Hospitalizations, and Deaths, by External Cause, United States, 2002-2006

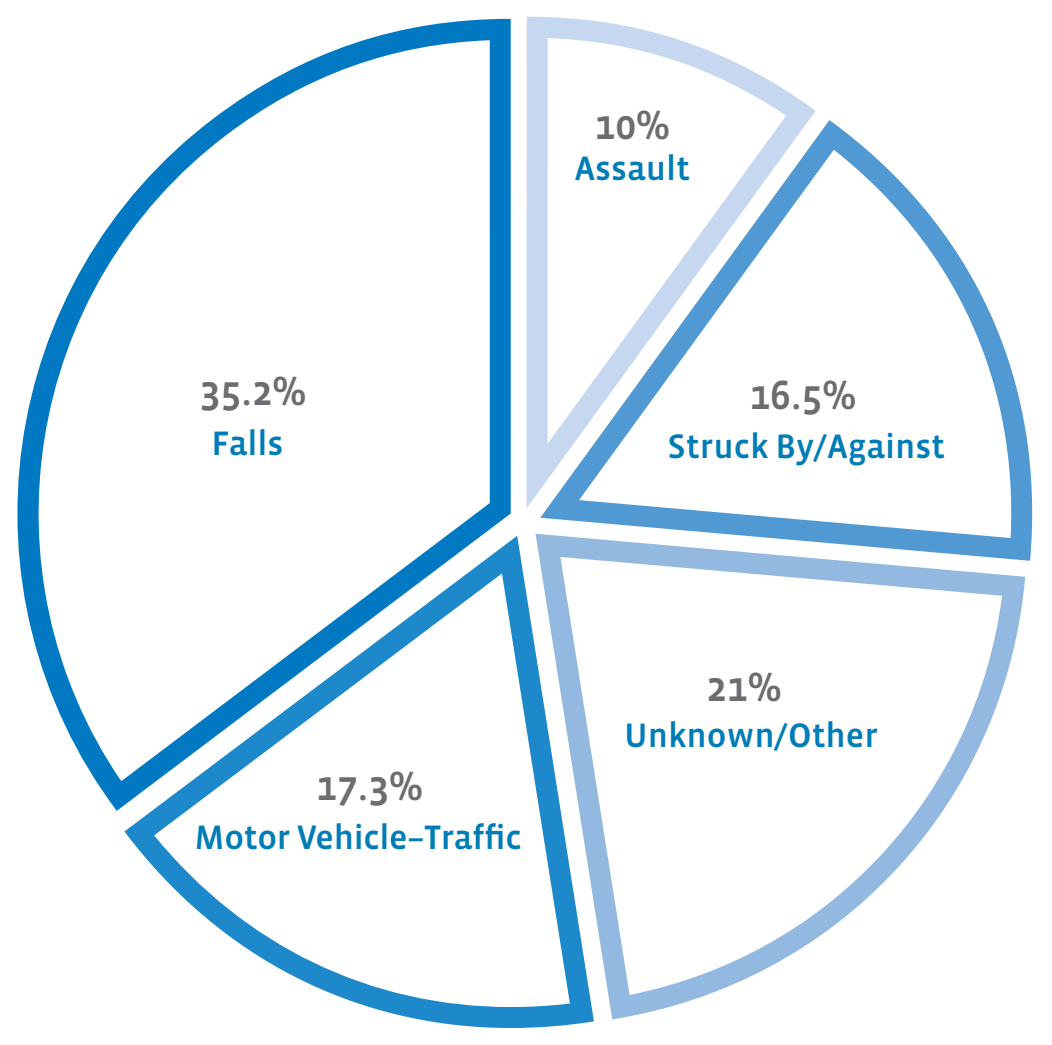




\section{TBI BY EXTERNAL CAUSE}

\section{COMPARING THE PERCENTAGES BY AGE GROUPS}

FIGURE 6: Estimated Average Percentage of Annual

Traumatic Brain Injury-Combined Emergency Department Visits, Hospitalizations, and Deaths Among Children o to

14 Years, by External Cause, United States, 2002-2006

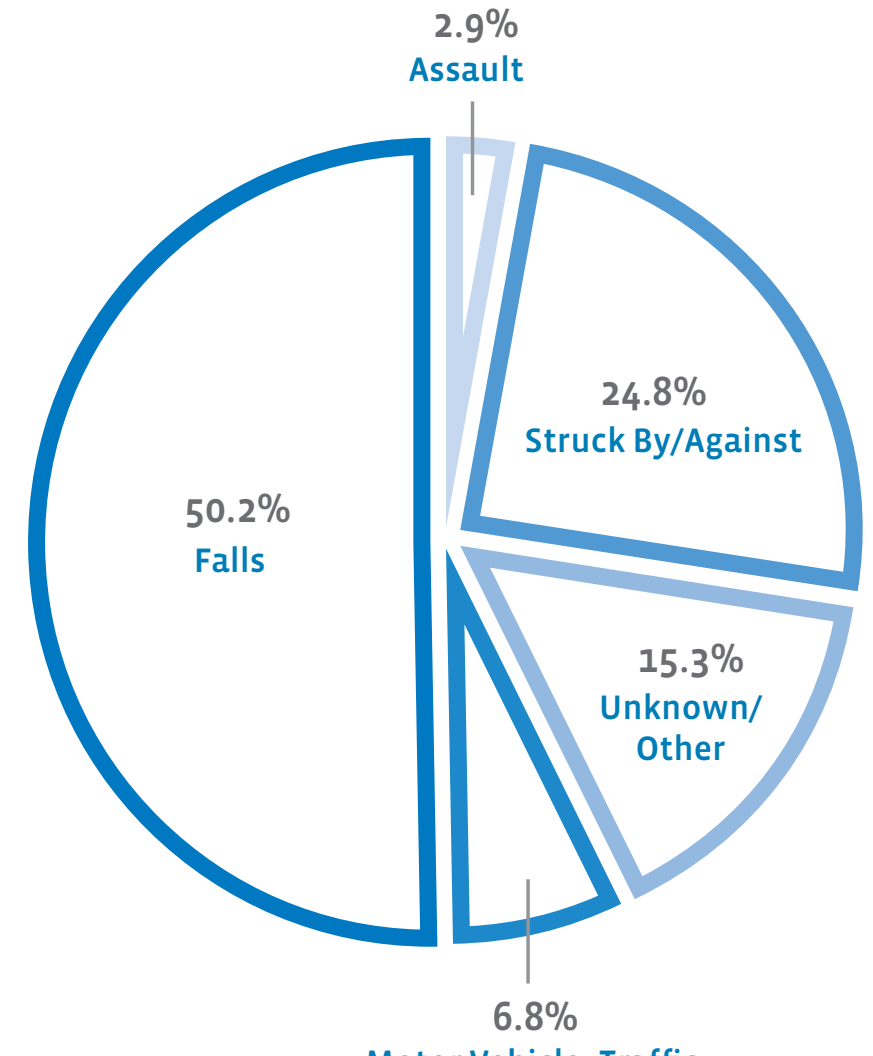

Motor Vehicle-Traffic
FIGURE 7: Estimated Average Percentage of Annual

Traumatic Brain Injury-Combined Emergency Department Visits, Hospitalizations, and Deaths Among Adults 65 Years and Older, by External Cause, United States, 2002-2006

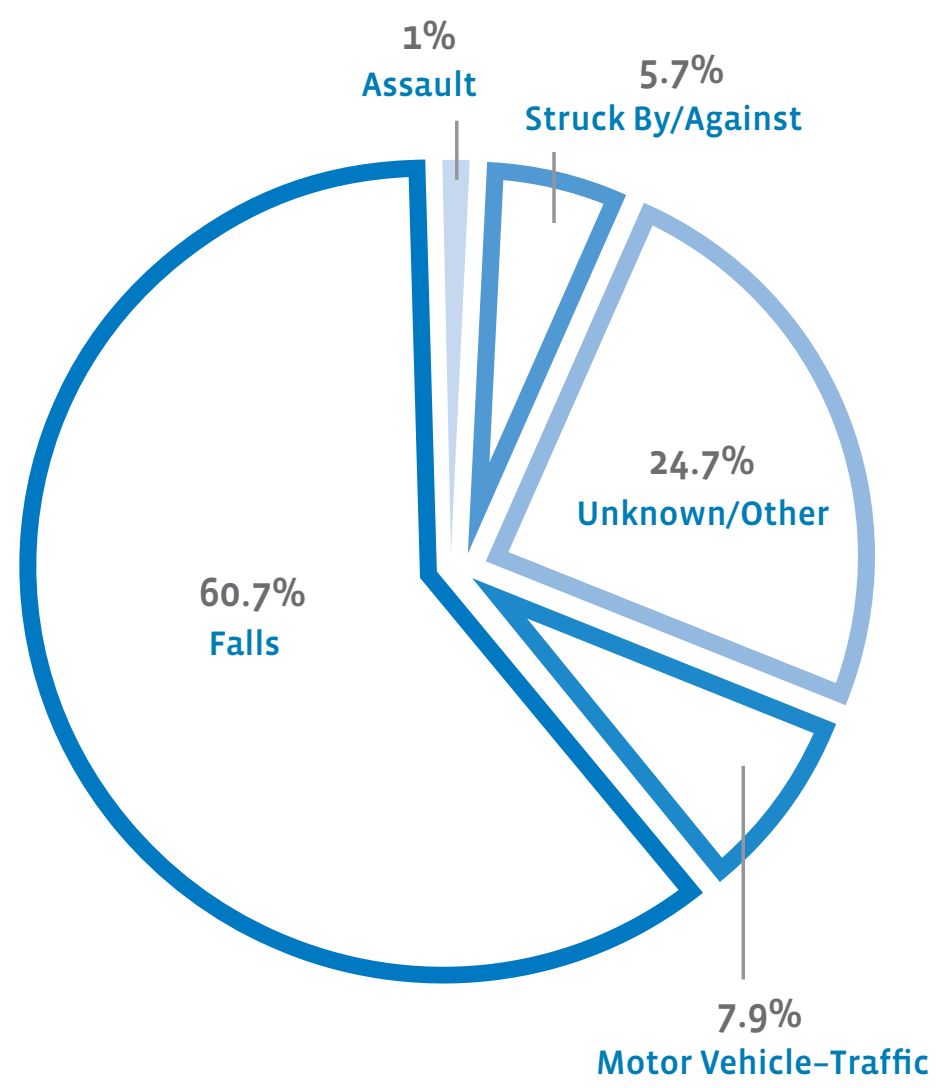




\section{SUMMARY OF FINDINGS BY EXTERNAL TBI CAUSES}

Falls

- Among all age groups, falls continued to be the leading cause of TBI (35.2\%). Falls cause approximately half $(50.2 \%)$ of the TBIs among children aged o to 14 years, compared with $60.7 \%$ among adults aged 65 years and older.

\section{Motor Vehicle-Traffic}

- Among all age groups, motor vehicle-traffic was the second leading cause of TBI (17.3\%) and resulted in the largest percentage of TBI-related deaths (31.8\%).

\section{Struck By/Against Events}

- Struck by/against events, which include colliding with a moving or stationary object, were the second leading cause of TBI among children aged 0 to 14 years $(24.8 \%)$.

\section{Assault}

- Assaults produced $10 \%$ of TBIs in the general population; they accounted for only $2.9 \%$ in children aged 0 to 14 years and $1 \%$ in adults aged 65 years old and older.

\section{CONCLUSION}

Presenting data on TBI is critical to understanding the impact of this important public health problem in the United States. This information provides the building blocks to inform TBI prevention strategies, identify research and education priorities, and support the need for services among those living with a TBI.

An estimated 1.7 million TBI-related emergency department visits, hospitalizations, and deaths occur each year in the United States. This is an increase from 1.4 million TBI-related emergency department visits, hospitalizations, and deaths from what was reported in the previous edition of Traumatic Brain Injury in the United States: Emergency Department Visits, Hospitalizations and Deaths, published by CDC in 2004. ${ }^{1}$ The increase in TBI-related injury was most apparent when examining emergency department visits. There were large increases in emergency department visits among children and older adults. Other major reasons for the increase were more fall-related TBIs and overall population growth. It is also likely that the public's awareness of TBI contributed to increased treatment. Further research and education is needed to explore how best to prevent traumatic brain injury.

Although this report provides data on a wide range of TBIs occurring in this country, it is not currently possible to capture all cases of TBI. There is no estimate for the number of people with non-fatal TBI seen outside of an emergency department of hospital or who receive no care at all. 
TABLE 1: Estimated Average Annual Numbers, Rates, and Percentages of Traumatic Brain Injury-Related Emergency Department Visits, Hospitalizations, and Deaths, by Age Group, United States, 2002-2006

\begin{tabular}{|c|c|c|c|c|c|c|c|c|c|c|c|}
\hline \multirow[b]{3}{*}{ AGE (YRS) } & \multicolumn{9}{|c|}{ DISPOSITION } & & \\
\hline & \multicolumn{3}{|c|}{ EMERGENCY DEPARTMENT VISITS" } & \multicolumn{3}{|c|}{ HOSPITALIZATIONS $^{\circ}$} & \multicolumn{3}{|c|}{ DEATHS* ${ }^{*}$} & \multicolumn{2}{|c|}{ TOTAL } \\
\hline & NUMBER & RATE & ROW \% & NUMBER & RATE & ROW \% & NUMBER & RATE & ROW \% & NUMBER & RATE' \\
\hline $0-4$ & 251,546 & 1256.2 & 93.9 & 15,239 & 76.1 & 5.7 & 998 & 5.0 & 0.4 & 267,783 & 1337.3 \\
\hline $5-9$ & 105,015 & 532.9 & 91.9 & 8,799 & 44.7 & 7.7 & 450 & 2.3 & 0.4 & 114,264 & 579.9 \\
\hline $10-14$ & 117,387 & 559.8 & 90.8 & 11,098 & 52.9 & 8.6 & 726 & 3.5 & 0.6 & 129,211 & 616.2 \\
\hline $15-19$ & 157,198 & 757.0 & 84.5 & 24,896 & 119.9 & 13.4 & 3,995 & 19.2 & 2.1 & 186,089 & 896.2 \\
\hline $20-24$ & 136,079 & 655.8 & 84.1 & 20,683 & 99.7 & 12.8 & 5,048 & 24.3 & 3.1 & 161,810 & 779.8 \\
\hline $25-34$ & 174,811 & 438.3 & 83.0 & 28,953 & 72.6 & 13.7 & 6,826 & 17.1 & 3.2 & 210,591 & 528.0 \\
\hline $35-44$ & 123,436 & 279.9 & 75.8 & 32,310 & 73.3 & 19.9 & 6,995 & 15.9 & 4.3 & 162,741 & 369.1 \\
\hline $45-54$ & 99,715 & 239.7 & 73.4 & 29,068 & 69.9 & 21.4 & 7,125 & 17.1 & 5.2 & 135,908 & 326.7 \\
\hline $55-64$ & 57,612 & 198.2 & 67.6 & 22,600 & 77.7 & 26.5 & 5,028 & 17.3 & 5.9 & 85,240 & 293.2 \\
\hline $65-74$ & 46,365 & 250.2 & 64.7 & 20,990 & 113.3 & 29.3 & 4,252 & 22.9 & 5.9 & 71,607 & 386.4 \\
\hline$\geq 75$ & 95,633 & 536.2 & 57.5 & 60,510 & 339.3 & 36.4 & 10,095 & 56.6 & 6.1 & 166,237 & 932.0 \\
\hline Total & $1,364,797$ & 465.4 & 80.7 & 275,146 & 93.8 & 16.3 & 51,538 & 17.6 & 3.0 & $1,691,481$ & 576.8 \\
\hline Adjusted? & & 468.0 & & & 93.6 & & & 17.4 & & & 579.0 \\
\hline
\end{tabular}

- Persons who were hospitalized, died, or transferred to another facility were excluded.

$\checkmark$ In-hospital deaths and patients who transferred from another hospital were excluded.

* 128 mortality records (2002-2006) were omitted because of missing age information.

- Average annual rate per 100,000 population.

g Age-adjusted to the 2000 U.S. standard population.

Numbers subject to rounding error. 
TABLE 2: Estimated Average Annual Numbers and Percentages of Traumatic Brain Injury-Related Emergency Department Visits, by Age Group and Disposition, United States, 2002-2006

\begin{tabular}{|c|c|c|c|c|c|}
\hline \multirow[b]{2}{*}{ AGE (YRS) } & \multicolumn{2}{|c|}{ TREATED AND RELEASED } & \multicolumn{2}{|c|}{ OTHER* } & \multirow{2}{*}{$\begin{array}{c}\text { TOTAL } \\
\text { NUMBER }\end{array}$} \\
\hline & NUMBER & ROW \% & NUMBER & ROW \% & \\
\hline $0-4$ & 251,546 & 92.9 & $19,106^{\dagger}$ & $7.1^{\dagger}$ & 270,652 \\
\hline $5-9$ & 105,015 & 86.1 & $16,940^{\S}$ & $13.9^{\S}$ & 121,955 \\
\hline $10-14$ & 117,387 & 92.4 & $9,617^{\S}$ & $7.6^{\S}$ & 127,004 \\
\hline $15-19$ & 157,198 & 88.6 & $20,131^{\dagger}$ & $11.4^{\dagger}$ & 177,329 \\
\hline $20-24$ & 136,079 & 85.6 & $22,949^{\dagger}$ & $14.4^{\dagger}$ & 159,028 \\
\hline $25-34$ & 174,811 & 92.1 & $15,005^{\dagger}$ & $7.9^{\dagger}$ & 189,816 \\
\hline $35-44$ & 123,436 & 83.8 & $23,936^{\dagger}$ & $16.2^{\dagger}$ & 147,372 \\
\hline $45-54$ & 99,715 & 78.8 & $26,898^{\dagger}$ & $21.2^{\dagger}$ & 126,613 \\
\hline $55-64$ & 57,612 & 74.6 & $19,623^{\S}$ & $25.4^{\S}$ & 77,235 \\
\hline $65-74$ & 46,365 & 78.9 & $12,394^{\S}$ & $21.1^{\S}$ & 58,759 \\
\hline$\geq 75$ & 95,633 & 66.3 & 48,681 & 33.7 & 144,314 \\
\hline Total & $1,364,797$ & 85.3 & 235,280 & 14.7 & $1,600,077$ \\
\hline
\end{tabular}

* Includes people who were hospitalized, died, or transferred to another facility. These records were excluded from the remaining emergency department tables.

† Sample size is 30-59; the value of the estimate was reported but may not be stable.

§ Sample size is less than 30 ; the value of the estimate was also reported, but it is not considered stable.

Numbers subject to rounding error. 
TABLE 3: Estimated Average Annual Numbers, Rates, and Percentages of Traumatic Brain Injury-Related Emergency Department Visits, by Age Group and Sex, United States, 2002-2006

\begin{tabular}{|c|c|c|c|c|c|c|c|c|}
\hline \multirow[b]{2}{*}{ AGE (YRS) } & \multicolumn{2}{|c|}{ MALE } & \multicolumn{4}{|c|}{ FEMALE } & \multicolumn{2}{|c|}{ TOTAL } \\
\hline & NUMBER & RATE & ROW \% & NUMBER & RATE" & ROW \% & NUMBER & RATE \\
\hline $0-4$ & 139,001 & 1357.4 & 55.3 & 112,545 & 1150.3 & 44.7 & 251,546 & 1256.2 \\
\hline $5-9$ & 68,671 & 681.2 & 65.4 & 36,343 & 377.6 & 34.6 & 105,014 & 532.9 \\
\hline $10-14$ & 90,221 & 840.0 & 76.9 & $27,166^{\dagger}$ & $265.6^{\dagger}$ & $23.1^{\dagger}$ & 117,387 & 559.8 \\
\hline $15-19$ & 98,761 & 926.6 & 62.8 & 58,437 & 578.2 & 37.2 & 157,198 & 757.0 \\
\hline $20-24$ & 86,669 & 812.2 & 63.7 & 49,410 & 490.3 & 36.3 & 136,079 & 655.8 \\
\hline $25-34$ & 97,845 & 483.8 & 56.0 & 76,966 & 391.5 & 44.0 & 174,811 & 438.3 \\
\hline $35-44$ & 68,527 & 311.4 & 55.5 & 54,909 & 248.6 & 44.5 & 123,436 & 279.9 \\
\hline $45-54$ & 50,941 & 249.2 & 51.1 & 48,775 & 230.5 & 48.9 & 99,716 & 239.7 \\
\hline $55-64$ & $32,226^{\dagger}$ & $230.2^{\dagger}$ & $55.9^{\dagger}$ & $25,386^{\dagger}$ & $168.4^{\dagger}$ & $44.1^{\dagger}$ & 57,612 & 198.2 \\
\hline $65-74$ & $23,146^{\dagger}$ & $273.7^{\dagger}$ & $49.9^{\dagger}$ & $23,218^{\dagger}$ & $230.5^{\dagger}$ & $50.1^{\dagger}$ & 46,364 & 250.2 \\
\hline$\geq 75$ & $33,917^{\dagger}$ & $504.4^{\dagger}$ & $35.5^{\dagger}$ & 61,716 & 555.4 & 64.5 & 95,633 & 536.2 \\
\hline Total & 789,925 & 547.6 & 57.9 & 574,871 & 385.9 & 42.1 & $1,364,796$ & 465.4 \\
\hline Adjusted ๆ & & 543.9 & & & 388.6 & & & 468.0 \\
\hline
\end{tabular}

- Average annual rate per 100,000 population.

+ Sample size is 30-59; the value of the estimate was reported but may not be stable.

I Age-adjusted to the 2000 U.S. standard population.

Note: Persons who were hospitalized, died, or transferred to another facility were excluded.

Numbers subject to rounding error. 
TABLE 4: Estimated Average Annual Numbers, Rates, and Percentages of Traumatic Brain Injury-Related Emergency Department Visits, by Age Group and Race, United States, 2002-2006

\begin{tabular}{|c|c|c|c|c|c|c|c|c|c|c|c|c|c|}
\hline \multirow[b]{2}{*}{ AGE (YRS) } & \multicolumn{3}{|c|}{ WHITE } & \multicolumn{3}{|c|}{ BLACK } & \multicolumn{3}{|c|}{$\begin{array}{l}\text { AMERICAN INDIAN, ALASKA NATIVE, } \\
\text { ASIAN, OR PACIFIC ISLANDER }\end{array}$} & \multicolumn{2}{|c|}{$\begin{array}{l}\text { OTHER/ } \\
\text { UNKNOWN }\end{array}$} & \multicolumn{2}{|c|}{ TOTAL } \\
\hline & NUMBER & RATE & ROW \% & NUMBER & RATE & ROW \% & NUMBER & RATE' & ROW \% & NUMBER & ROW \% & NUMBER & RATE \\
\hline $0-4$ & 180,880 & 1160.8 & 71.9 & 56,942 & 1746.6 & 22.6 & $13,576^{\dagger}$ & $1148.5^{\dagger}$ & $5.4^{\dagger}$ & $147^{\S}$ & $0.1^{\S}$ & 251,545 & 1256.2 \\
\hline $5-9$ & 76,588 & 499.5 & 72.9 & $22,994^{\dagger}$ & $718.5^{\dagger}$ & $21.9^{\dagger}$ & $5,433^{\S}$ & $463.1^{\S}$ & $5.2^{\S}$ & -- & -- & 105,015 & 532.9 \\
\hline $10-14$ & 84,096 & 517.2 & 71.6 & $27,156^{\dagger}$ & $775.0^{\dagger}$ & $23.1^{\dagger}$ & $5,509^{\S}$ & $456.5^{\S}$ & $4.7^{\S}$ & $625^{\S}$ & $0.5^{\S}$ & 117,386 & 559.8 \\
\hline $15-19$ & 128,896 & 793.1 & 82.0 & $23,049^{\dagger}$ & $696.5^{\dagger}$ & $14.7^{\dagger}$ & $4,586^{\S}$ & $380.8^{\S}$ & $2.9^{\S}$ & $666^{\S}$ & $0.4^{\S}$ & 157,197 & 757.0 \\
\hline $20-24$ & 105,796 & 647.4 & 77.7 & $27,030^{\dagger}$ & $867.5^{\dagger}$ & $19.9^{\dagger}$ & $3,253^{\S}$ & $251.9^{\S}$ & $2.4^{\S}$ & -- & -- & 136,079 & 655.8 \\
\hline $25-34$ & 137,732 & 438.3 & 78.8 & $29,968^{\dagger}$ & $542.9^{\dagger}$ & $17.1^{\dagger}$ & $6,445^{\S}$ & $218.9^{\S}$ & $3.7^{\S}$ & $666^{\S}$ & $0.4^{\S}$ & 174,811 & 438.3 \\
\hline $35-44$ & 103,176 & 289.7 & 83.6 & $14,158^{\dagger}$ & $247.0^{\dagger}$ & $11.5^{\dagger}$ & $5,767^{\S}$ & $209.9^{\S}$ & $4.7^{\S}$ & $334^{\S}$ & $0.3^{\S}$ & 123,435 & 279.9 \\
\hline $45-54$ & 76,966 & 223.4 & 77.2 & $20,040^{\dagger}$ & $407.3^{\dagger}$ & $20.1^{\dagger}$ & $2,710^{\S}$ & $121.2^{\S}$ & $2.7^{\S}$ & -- & -- & 99,716 & 239.7 \\
\hline $55-64$ & 47,644 & 192.2 & 82.7 & $7,385^{\S}$ & $253.7^{\S}$ & $12.8^{\S}$ & $2,584^{\S}$ & $187.9^{\S}$ & $4.5^{\S}$ & -- & -- & 57,613 & 198.2 \\
\hline $65-74$ & 36,979 & 230.9 & 79.8 & $5,236^{\S}$ & $300.9^{\S}$ & $11.3^{\S}$ & $3,196^{\S}$ & $411.4^{\S}$ & $6.9^{\S}$ & $954^{\S}$ & $2.1^{\S}$ & 46,365 & 250.2 \\
\hline$\geq 75$ & 88,260 & 553.4 & 92.3 & $4,626^{\S}$ & $342.0^{\S}$ & $4.8^{\S}$ & $2,748^{\S}$ & $512.6^{\S}$ & $2.9^{\S}$ & -- & -- & 95,634 & 536.2 \\
\hline Total & $1,067,013$ & 448.3 & 78.2 & 238,584 & 618.6 & 17.5 & 55,807 & 334.7 & 4.1 & 3,392 & 0.2 & $1,364,796$ & 465.4 \\
\hline Adjusted ? & & 456.6 & & & 568.7 & & & 345.2 & & & & & 468.0 \\
\hline
\end{tabular}

- Average annual rate per 100,000 population.

† Sample size is 30-59; the value of the estimate was reported but may not be stable.

§ Sample size is less than 30; the value of the estimate was also reported, but it is not considered stable.

If Age-adjusted to the 2000 U.S. standard population.

-- No data for these cells.

Note: Persons who were hospitalized, died, or transferred to another facility were excluded.

Numbers subject to rounding error. 
TABLE 5: Estimated Average Annual Numbers, Rates, and Percentages of Traumatic Brain Injury-Related Emergency Department Visits, by Age Group and External Cause, United States, 2002-2006

\begin{tabular}{|c|c|c|c|c|c|c|c|c|c|c|c|c|c|c|c|c|c|}
\hline \multirow[b]{2}{*}{ AGE (YRS) } & \multicolumn{3}{|c|}{$\begin{array}{l}\text { MOTOR VEHICLE- } \\
\text { TRAFFIC* }\end{array}$} & \multicolumn{3}{|c|}{ FALLS } & \multicolumn{3}{|c|}{ ASSAULT } & \multicolumn{3}{|c|}{ STRUCK BY/AGAINST } & \multicolumn{3}{|c|}{ OTHER/UNKNOWN } & \multicolumn{2}{|c|}{ TOTAL } \\
\hline & NUMBER & RATE & ROW \% & 6 NUMBER & RATE' & ROW \% & NUMBER & RATE & ROW \% & NUMBER & RATE" & ROW \% & NUMBER & RATE & ROW \% & NUMBER & RATE \\
\hline $0-4$ & $12,852^{\S}$ & $64.2^{\S}$ & $5.1^{\S}$ & 161,455 & 806.3 & 64.2 & $362^{\S}$ & $1.8^{\S}$ & $0.1^{\S}$ & 53,922 & $\overline{269.3}$ & 21.4 & $22,954^{\dagger}$ & $114.6^{\dagger}$ & $9.1^{\dagger}$ & 251,545 & 1256.2 \\
\hline $5-9$ & $7,310^{\S}$ & $37.1^{\S}$ & $7.0^{\S}$ & 42,371 & 215.0 & 40.3 & $1,033^{\S}$ & $5.2^{\S}$ & $1.0^{\S}$ & $35,583^{\dagger}$ & $180.6^{\dagger}$ & $33.9^{\dagger}$ & $18,718^{\dagger}$ & $95.0^{\dagger}$ & $17.8^{\dagger}$ & 105,015 & 532.9 \\
\hline $10-14$ & $6,529^{\S}$ & $31.1^{\S}$ & $5.6^{\S}$ & $42,843^{\dagger}$ & $204.3^{\dagger}$ & $36.5^{\dagger}$ & $11,385^{\S}$ & $54.3^{\S}$ & $9.7^{\S}$ & 34,572 & 164.9 & 29.5 & $22,057^{\dagger}$ & $105.2^{\dagger}$ & $18.8^{\dagger}$ & 117,386 & 559.8 \\
\hline $15-19$ & 40,466 & 194.9 & 25.7 & $32,740^{\dagger}$ & $157.7^{\dagger}$ & 20.8 & $22,272^{\dagger}$ & $107.3^{\dagger}$ & $14.2^{\dagger}$ & 36,512 & 175.8 & 23.2 & $25,207^{\dagger}$ & $121.4^{\dagger}$ & $16.0^{\dagger}$ & 157,197 & 757.0 \\
\hline $20-24$ & 44,209 & 213.1 & 32.5 & $19,845^{\dagger}$ & $95.6^{\dagger}$ & $14.6^{\dagger}$ & $33,360^{\dagger}$ & $160.8^{\dagger}$ & $24.5^{\dagger}$ & $19,205^{\S}$ & $92.6^{\S}$ & $14.1^{\S}$ & $19,459^{\S}$ & $93.8^{\S}$ & $14.3^{\S}$ & 136,078 & 655.8 \\
\hline $25-34$ & $42,213^{\dagger}$ & $105.8^{\dagger}$ & $24.1^{\dagger}$ & $31,794^{\dagger}$ & $79.7^{\dagger}$ & $18.2^{\dagger}$ & $36,385^{\dagger}$ & $91.2^{\dagger}$ & $20.8^{\dagger}$ & $30,464^{\dagger}$ & $76.4^{\dagger}$ & $17.4^{\dagger}$ & $33,954^{\dagger}$ & $85.1^{\dagger}$ & $19.4^{\dagger}$ & 174,810 & 438.3 \\
\hline $35-44$ & $19,687^{\dagger}$ & $44.6^{\dagger}$ & $15.9^{\dagger}$ & $34,510^{\dagger}$ & $78.3^{\dagger}$ & $28.0^{+}$ & $20,775^{\dagger}$ & $47.1^{\dagger}$ & $16.8^{\dagger}$ & $21,689^{\dagger}$ & $49.2^{\dagger}$ & $17.6^{\dagger}$ & $26,776^{+}$ & $60.7^{\dagger}$ & $21.7^{\dagger}$ & 123,437 & 279.9 \\
\hline $45-54$ & $20,210^{\dagger}$ & $48.6^{\dagger}$ & $20.3^{\dagger}$ & $33,779^{\dagger}$ & $81.2^{\dagger}$ & $33.9^{\dagger}$ & $14,610^{\S}$ & $35.1^{\S}$ & $14.7^{\S}$ & $17,216^{\S}$ & $41.4^{\S}$ & $17.3^{\S}$ & $13,900^{\S}$ & $33.4^{\S}$ & $13.9^{\S}$ & 99,715 & 239.7 \\
\hline $55-64$ & $14,454^{\S}$ & $49.7^{\S}$ & $25.1^{\S}$ & $16,485^{\dagger}$ & $56.7^{\dagger}$ & $28.6^{\dagger}$ & $6,548^{\S}$ & $22.5^{\S}$ & $11.4^{\S}$ & $10,015^{\S}$ & $34.4^{\S}$ & $17.4^{\S}$ & $10,110^{\S}$ & $34.8^{\S}$ & $17.5^{\S}$ & 57,612 & 198.2 \\
\hline $65-74$ & $5,904^{\S}$ & $31.9^{\S}$ & $12.7^{\S}$ & $28,698^{\dagger}$ & $154.9^{\dagger}$ & $61.9^{\dagger}$ & $1,329^{\S}$ & $7.2^{\S}$ & $2.9^{\S}$ & $7,123^{\S}$ & $38.4^{\S}$ & $15.4^{\S}$ & $3,311^{\S}$ & $17.9^{\S}$ & $7.1^{\S}$ & 46,365 & 250.2 \\
\hline$\geq 75$ & $5,102^{\S}$ & $28.6^{\S}$ & $5.3^{\S}$ & 78,523 & 440.2 & 82.1 & $411^{\S}$ & $2.3^{\S}$ & $0.4^{\S}$ & $5,412^{\S}$ & $30.3^{\S}$ & $5.7^{\S}$ & $6,185^{\S}$ & $34.7^{\S}$ & $6.5^{\S}$ & 95,633 & 536.2 \\
\hline Total & 8,936 & 74.7 & 16.0 & 3,043 & 178.4 & 38.3 & 148,470 & 50.6 & 10.9 & 271,713 & 92.7 & 19.9 & 202,631 & 69.1 & 14. & 93 & 465.4 \\
\hline Adjusted ๆ & & 74.0 & & & 180.2 & & & 50.2 & & & 93.9 & & & 69.7 & & & 468.0 \\
\hline
\end{tabular}

* Motor vehicle-traffic includes the following external cause of injury: occupant, motorcyclist, pedal cyclist, pedestrian, other and unspecified person involved in a motor vehicle-traffic incident.

- Average annual rate per 100,000 population.

+ Sample size is 30-59; the value of the estimate was reported but may not be stable.
§ Sample size is less than 30; the value of the estimate was also reported, but it is not considered stable.

I Age-adjusted to the 2000 U.S. standard population.

Note: Persons who were hospitalized, died, or transferred to another facility were excluded.

Numbers subject to rounding error. 
TABLE 6: Estimated Average Annual Numbers, Rates, and Percentages of Traumatic Brain Injury-Related Emergency Department Visits, by Age Group and Specific Motor Vehicle-Traffic (MVT) External Causes, United States, 2002-2006

\begin{tabular}{|c|c|c|c|c|c|c|c|c|c|c|c|c|c|c|c|c|c|}
\hline \multirow[b]{2}{*}{ AGE (YRS) } & \multicolumn{3}{|c|}{ MVT-OCCUPANT } & \multicolumn{3}{|c|}{ MVT- MOTORCYCLE } & \multicolumn{3}{|c|}{ MVT- PEDAL CYCLE } & \multicolumn{3}{|c|}{ MVT- PEDESTRIAN } & \multicolumn{3}{|c|}{$\begin{array}{c}\text { MVT- OTHER } \\
\text { OR UNSPECIFIED }\end{array}$} & \multicolumn{2}{|c|}{ TOTAL } \\
\hline & NUMBER & RATE' & ROW \% & NUMBER & RATE' & ROW \% & NUMBER & RATE' & ROW \% & NUMBER & RATE & ROW \% & NUMBER & RATE' & ROW \% & NUMBER & RATE \\
\hline $0-4$ & $9,498^{\S}$ & $\overline{47.4^{\S}}$ & $73.9^{\S}$ & -- & -- & -- & -- & -- & -- & -- & -- & -- & $3,355^{\S}$ & $16.8^{\S}$ & $26.1^{\S}$ & $12,853^{\S}$ & $64.2^{\S}$ \\
\hline $5-9$ & $1,427 \S$ & $7.2^{\S}$ & $19.5^{\S}$ & -- & -- & -- & $513^{\S}$ & $2.6^{\S}$ & $7.0^{\S}$ & $514^{\S}$ & $2.6^{\S}$ & $7.0^{\S}$ & $4,856^{\S}$ & $24.6^{\S}$ & $66.4^{\S}$ & $7,310^{\S}$ & $37.1^{\S}$ \\
\hline $10-14$ & $2,455^{\S}$ & $11.7^{\S}$ & $37.6^{\S}$ & $787^{\S}$ & $3.8^{\S}$ & $12.1^{\S}$ & -- & -- & -- & $2,959^{\S}$ & $14.1^{\S}$ & $45.3^{\S}$ & $328^{\S}$ & $1.6^{\S}$ & $5.0^{\S}$ & $6,529^{\S}$ & $31.1^{\S}$ \\
\hline $15-19$ & $18,828^{\dagger}$ & $90.7^{\dagger}$ & $46.5^{\dagger}$ & $568^{\S}$ & $2.7^{\S}$ & $1.4^{\S}$ & -- & -- & -- & $324^{\S}$ & $1.6^{\S}$ & $0.8^{\S}$ & $20,745^{\S}$ & $99.9^{\S}$ & $51.3^{\S}$ & 40,465 & 194.9 \\
\hline $20-24$ & $24,985^{\dagger}$ & $120.4^{\dagger}$ & $56.5^{\dagger}$ & $3,110^{\S}$ & $15.0^{\S}$ & $7.0^{\S}$ & $37 \S$ & $0.2^{\S}$ & $0.1^{\S}$ & $1,420^{\S}$ & $6.8^{\S}$ & $3.2^{\S}$ & $14,657^{\S}$ & $70.6^{\S}$ & $33.2^{\S}$ & 44,209 & 213.1 \\
\hline $25-34$ & $18,786^{\S}$ & $47.1^{\S}$ & $44.5^{\S}$ & $4,847^{\S}$ & $12.2^{\S}$ & $11.5^{\S}$ & $563^{\S}$ & $1.4^{\S}$ & $1.3^{\S}$ & -- & -- & -- & $18,017^{\S}$ & $45.2^{\S}$ & $42.7^{\S}$ & $42,213^{\dagger}$ & $105.8^{\dagger}$ \\
\hline $35-44$ & $6,632^{\S}$ & $15.0^{\S}$ & $33.7^{\S}$ & $189^{\S}$ & $0.4^{\S}$ & $1.0^{\S}$ & -- & -- & -- & $307 \S$ & $0.7^{\S}$ & $1.6^{\S}$ & $12,559 \S$ & $28.5^{\S}$ & $63.8^{\S}$ & $19,687^{\dagger}$ & $44.6^{\dagger}$ \\
\hline $45-54$ & $12,616^{\S}$ & $30.3^{\S}$ & $62.4^{\S}$ & $304^{\S}$ & $0.7^{\S}$ & $1.5^{\S}$ & -- & -- & -- & $1,233^{\S}$ & $3.0^{\S}$ & $6.1^{\S}$ & $6,057^{\S}$ & $14.6^{\S}$ & $30.0^{\S}$ & $20,210^{\dagger}$ & $48.6^{\dagger}$ \\
\hline $55-64$ & $6,450^{\S}$ & $22.2^{\S}$ & $44.6^{\S}$ & $133^{\S}$ & $0.5^{\S}$ & $0.9^{\S}$ & -- & -- & -- & $169^{\S}$ & $0.6^{\S}$ & $1.2^{\S}$ & $7,701^{\S}$ & $26.5^{\S}$ & $53.3^{\S}$ & $14,453^{\S}$ & $49.7 \S$ \\
\hline $65-74$ & $2,689 \S$ & $14.5^{\S}$ & $45.6^{\S}$ & -- & -- & -- & -- & -- & -- & $1,000^{\S}$ & $5.4^{\S}$ & $16.9^{\S}$ & $2,214^{\S}$ & $12.0^{\S}$ & $37.5^{\S}$ & $5,903^{\S}$ & $31.9^{\S}$ \\
\hline$\geq 75$ & -- & -- & -- & -- & -- & -- & -- & -- & -- & -- & -- & -- & $5,102^{\S}$ & $28.6^{\S}$ & $100.0^{\S}$ & $5,102^{\S}$ & $28.6^{\S}$ \\
\hline Total & 104,366 & 35.6 & 47.7 & $9,938^{\S}$ & $3.4^{\S}$ & $4.5^{\S}$ & $1,113^{\S}$ & $0.4^{\S}$ & $0.5^{\S}$ & $7,926^{\S}$ & $2.7^{\S}$ & $3.6^{\S}$ & 95,591 & 32.6 & 43.7 & 218,934 & 74.7 \\
\hline Adjusted ? & & 35.0 & & & 3.3 & & & 0.4 & & & 2.7 & & & 32.5 & & & 74.0 \\
\hline
\end{tabular}

- Average annual rate per 100,000 population.

† Sample size is 30-59; the value of the estimate was reported but may not be stable.

§ Sample size is less than 30; the value of the estimate was also reported, but it is not considered stable.

T) Age-adjusted to the 2000 U.S. standard population.

-- No data for these cells.
Note: Persons who were hospitalized, died, or transferred to another facility were excluded.

Numbers subject to rounding error. 
TABLE 7: Estimated Average Annual Numbers and Percentages of Traumatic Brain Injury-Related Emergency Department Visits, by Age Group and Expected Source of Payment, United States, 2002-2006

\begin{tabular}{|c|c|c|c|c|c|c|c|c|c|c|c|}
\hline \multirow[b]{2}{*}{ AGE(YRS) } & \multicolumn{2}{|c|}{ PRIVATE } & \multicolumn{2}{|c|}{ MEDICAID } & \multicolumn{2}{|c|}{ MEDICARE } & \multicolumn{2}{|c|}{ WORKER'S COMPENSATION } & \multicolumn{2}{|c|}{ OTHER/UNKNOWN* } & \multirow{2}{*}{$\begin{array}{c}\text { TOTAL } \\
\text { NUMBER }\end{array}$} \\
\hline & NUMBER & ROW \% & NUMBER & ROW \% & NUMBER & ROW $\%$ & NUMBER & ROW \% & NUMBER & ROW \% & \\
\hline $0-4$ & 121,610 & 48.3 & 87,911 & 34.9 & $1,025^{\S}$ & $0.4^{\S}$ & -- & -- & $40,999^{\dagger}$ & $16.3^{\dagger}$ & 251,545 \\
\hline $5-9$ & 58,430 & 55.6 & $23,720^{\dagger}$ & $22.6^{\dagger}$ & $797^{\S}$ & $0.8^{\S}$ & -- & -- & $22,068^{\dagger}$ & $21.0^{\dagger}$ & 105,015 \\
\hline $10-14$ & 74,107 & 63.1 & $24,978^{\dagger}$ & $21.3^{\dagger}$ & $670^{\S}$ & $0.6^{\S}$ & -- & -- & $17,631^{\dagger}$ & $15.0^{\dagger}$ & 117,386 \\
\hline $15-19$ & 91,028 & 57.9 & $14,309^{\dagger}$ & $9.1^{\dagger}$ & $3,190^{\S}$ & $2.0^{\S}$ & $695^{\S}$ & $0.4^{\S}$ & 47,975 & 30.5 & 157,197 \\
\hline $20-24$ & 52,094 & 38.3 & $5,093^{\S}$ & $3.7^{\S}$ & -- & -- & $8,737^{\S}$ & $6.4^{\S}$ & 70,154 & 51.6 & 136,078 \\
\hline $25-34$ & 73,054 & 41.8 & $17,148^{\dagger}$ & $9.8^{\dagger}$ & $2,348^{\S}$ & $1.3^{\S}$ & $12,895^{\S}$ & $7.4^{\S}$ & 69,366 & 39.7 & 174,811 \\
\hline $35-44$ & 54,807 & 44.4 & $16,854^{\dagger}$ & $13.7^{\dagger}$ & $7,118^{\S}$ & $5.8^{\S}$ & $7,542^{\S}$ & $6.1^{\S}$ & 37,114 & 30.1 & 123,435 \\
\hline $45-54$ & 46,457 & 46.6 & $12,355^{\S}$ & $12.4^{\S}$ & $4,426^{\S}$ & $4.4^{\S}$ & $6,219^{\S}$ & $6.2^{\S}$ & $30,259^{\dagger}$ & $30.3^{\dagger}$ & 99,716 \\
\hline $55-64$ & $24,566^{\dagger}$ & $42.6^{\dagger}$ & $6,412^{\S}$ & $11.1^{\S}$ & $3,802^{\S}$ & $6.6^{\S}$ & $4,201^{\S}$ & $7.3^{\S}$ & $18,631^{\S}$ & $32.3^{\S}$ & 57,612 \\
\hline $65-74$ & $6,558^{\S}$ & $14.1^{\S}$ & $3,424^{\S}$ & $7.4^{\S}$ & $29,249^{\dagger}$ & $63.1^{\dagger}$ & $1,773^{\S}$ & $3.8^{\S}$ & $5,361^{\S}$ & $11.6^{\S}$ & 46,365 \\
\hline$\geq 75$ & $5,636^{\S}$ & $5.9^{\S}$ & $10,461^{\S}$ & $10.9^{\S}$ & 70,994 & 74.2 & $1,571^{\S}$ & $1.6^{\S}$ & $6,971^{\S}$ & $7.3^{\S}$ & 95,633 \\
\hline Total & 608,347 & 44.6 & 222,665 & 16.3 & 123,619 & 9.1 & 43,633 & 3.2 & 366,529 & 26.9 & $1,364,793$ \\
\hline
\end{tabular}

* Includes self pay, no charge, other government, other, and unknown.

+ Sample size is 30-59; the value of the estimate was reported but may not be stable.

§ Sample size is less than 30; the value of the estimate was also reported, but it is not considered stable.

-- No data for these cells.

Note: Persons who were hospitalized, died, or transferred to another facility were excluded.

Numbers subject to rounding error. 
TABLE 8: Estimated Average Annual Numbers and Percentages of Traumatic Brain Injury-Related Hospitalizations, by Age Group and Disposition, United States, 2002-2006

\begin{tabular}{|c|c|c|c|c|c|c|c|c|c|}
\hline \multirow[b]{3}{*}{ AGE (YRS) } & \multicolumn{6}{|c|}{ DISCHARGED ALIVE ${ }^{\ddagger}$} & & & \multirow{3}{*}{$\begin{array}{r}\text { TOTAL } \\
\text { NUMBE }\end{array}$} \\
\hline & \multicolumn{2}{|c|}{ HOME } & \multicolumn{2}{|c|}{ TRANSFERRED* } & \multicolumn{2}{|c|}{ OTHER/UNKNOWN $^{+}$} & \multicolumn{2}{|c|}{ IN-HOSPITAL DEATHS ${ }^{\ddagger}$} & \\
\hline & NUMBER & ROW \% & NUMBER & ROW \% & NUMBER & ROW \% & NUMBER & ROW \% & \\
\hline $0-4$ & 15,564 & 87.8 & $855^{\S}$ & $4.8^{\S}$ & $903^{\dagger}$ & $5.1^{\dagger}$ & $406^{\S}$ & $2.3^{\S}$ & 17,728 \\
\hline $5-9$ & 8,996 & 89.8 & $283^{\S}$ & $2.8^{\S}$ & $281^{\S}$ & $2.8^{\S}$ & $461^{\S}$ & $4.6^{\S}$ & 10,021 \\
\hline $10-14$ & 10,855 & 89.4 & $326^{\S}$ & $2.7^{\S}$ & $429^{\dagger}$ & $3.5^{\dagger}$ & $535^{\S}$ & $4.4^{\S}$ & 12,145 \\
\hline $15-19$ & 21,971 & 78.9 & $2,064^{\dagger}$ & $7.4^{\dagger}$ & 2,459 & 8.8 & $1,356^{\dagger}$ & $4.9^{\dagger}$ & 27,850 \\
\hline $20-24$ & 18,261 & 79.8 & $1,567^{\dagger}$ & $6.9^{\dagger}$ & 1,776 & 7.8 & $1,277^{\dagger}$ & $5.6^{\dagger}$ & 22,881 \\
\hline $25-34$ & 23,239 & 75.7 & 1,976 & 6.4 & 4,295 & 14.0 & $1,199^{\dagger}$ & $3.9^{\dagger}$ & 30,709 \\
\hline $35-44$ & 25,192 & 73.9 & 3,154 & 9.3 & 4,864 & 14.3 & $881^{\dagger}$ & $2.6^{\dagger}$ & 34,091 \\
\hline $45-54$ & 23,042 & 72.4 & 3,166 & 9.9 & 3,782 & 11.9 & 1,827 & 5.7 & 31,817 \\
\hline $55-64$ & 16,359 & 64.6 & 4,223 & 16.7 & 3,112 & 12.3 & $1,617^{\dagger}$ & $6.4^{\dagger}$ & 25,311 \\
\hline $65-74$ & 13,331 & 55.7 & 5,670 & 23.7 & 2,969 & 12.4 & 1,983 & 8.3 & 23,953 \\
\hline$\geq 75$ & 24,751 & 34.7 & 30,302 & 42.4 & 9,453 & 13.2 & 6,926 & 9.7 & 71,432 \\
\hline Total & 201,561 & 65.5 & 53,586 & 17.4 & 34,323 & 11.1 & 18,468 & 6.0 & 307,938 \\
\hline
\end{tabular}

+ Includes patients who left against medical advice and who were discharged alive (but no disposition stated), and patients with unknown disposition.

* Includes both long- and short-term care facilities.

₹ In-hospital deaths and patients who transferred from another hospital were excluded from the remaining hospitalization tables. + Sample size is 30-59; the value of the estimate was reported but may not be stable.

$\S$ Sample size is less than 30 ; the value of the estimate was also reported, but is not considered stable.

Numbers subject to rounding error. 
TABLE 9: Estimated Average Annual Numbers, Rates, and Percentages of Traumatic Brain Injury-Related Hospitalizations, by Age Group and Sex, United States, 2002-2006

\begin{tabular}{|c|c|c|c|c|c|c|c|c|}
\hline \multirow[b]{2}{*}{ AGE (YRS) } & \multicolumn{3}{|c|}{ MALE } & \multicolumn{3}{|c|}{ FEMALE } & \multicolumn{2}{|c|}{ TOTAL } \\
\hline & NUMBER & RATE" & ROW \% & NUMBER & RATE» & ROW \% & NUMBER & RATE" \\
\hline $0-4$ & 9,019 & 88.1 & 59.2 & 6,220 & 63.6 & 40.8 & 15,239 & 76.1 \\
\hline $5-9$ & 5,296 & 52.5 & 60.2 & 3,503 & 36.4 & 39.8 & 8,799 & 44.7 \\
\hline $10-14$ & 7,407 & 69.0 & 66.7 & 3,691 & 36.1 & 33.3 & 11,098 & 52.9 \\
\hline $15-19$ & 17,189 & 161.3 & 69.0 & 7,708 & 76.3 & 31.0 & 24,897 & 119.9 \\
\hline $20-24$ & 16,341 & 153.1 & 79.0 & 4,343 & 43.1 & 21.0 & 20,684 & 99.7 \\
\hline $25-34$ & 22,438 & 110.9 & 77.5 & 6,516 & 33.1 & 22.5 & 28,954 & 72.6 \\
\hline $35-44$ & 22,417 & 101.9 & 69.4 & 9,893 & 44.8 & 30.6 & 32,310 & 73.3 \\
\hline $45-54$ & 20,085 & 98.2 & 69.1 & 8,983 & 42.5 & 30.9 & 29,068 & 69.9 \\
\hline $55-64$ & 15,269 & 109.1 & 67.6 & 7,331 & 48.6 & 32.4 & 22,600 & 77.7 \\
\hline $65-74$ & 11,437 & 135.2 & 54.5 & 9,553 & 94.8 & 45.5 & 20,990 & 113.3 \\
\hline$\geq 75$ & 23,360 & 347.4 & 38.6 & 37,150 & 334.3 & 61.4 & 60,510 & 339.3 \\
\hline Total & 170,258 & 118.0 & 61.9 & 104,891 & 70.4 & 38.1 & 275,149 & 93.8 \\
\hline Adjusted ๆ & & 121.0 & & & 66.2 & & & 93.6 \\
\hline
\end{tabular}

- Average annual rate per 100,000 population.

If Age-adjusted to the 2000 U.S. standard population.

Note: In-hospital deaths and patients who transferred from another hospital were excluded.

Numbers subject to rounding error. 
TABLE 10: Estimated Average Annual Numbers, Rates, and Percentages of Traumatic Brain InjuryRelated Hospitalizations, by Age Group and Race, United States, 2002-2006

\begin{tabular}{|c|c|c|c|c|c|c|c|c|c|c|c|c|c|}
\hline \multirow[b]{2}{*}{ AGE (YRS) } & \multicolumn{3}{|c|}{ WHITE } & \multicolumn{3}{|c|}{ BLACK } & \multicolumn{3}{|c|}{$\begin{array}{l}\text { AMERICAN INDIAN, ALASKA NATIVE, } \\
\text { ASIAN, OR PACIFIC ISLANDER }\end{array}$} & \multicolumn{2}{|c|}{$\begin{array}{l}\text { OTHER/ } \\
\text { UNKNOWN }\end{array}$} & \multicolumn{2}{|c|}{ TOTAL } \\
\hline & NUMBER & RATE & ROW \% & NUMBER & RATE' & ROW \% & NUMBER & RATE' & ROW \% & NUMBER & ROW \% & NUMBER & RATE" \\
\hline $0-4$ & 9,361 & 60.1 & 61.4 & 2,075 & 63.6 & 13.6 & $530^{\S}$ & $44.8^{\S}$ & $3.5^{\S}$ & 3,274 & 21.5 & 15,240 & 76.1 \\
\hline $5-9$ & 5,412 & 35.3 & 61.5 & 1,126 & 35.2 & 12.8 & $151^{\S}$ & $12.9^{\S}$ & $1.7^{\S}$ & 2,110 & 24.0 & 8,799 & 44.7 \\
\hline $10-14$ & 6,278 & 38.6 & 56.6 & 1,669 & 47.6 & 15.0 & $388^{\S}$ & $32.2^{\S}$ & $3.5^{\S}$ & 2,763 & 24.9 & 11,098 & 52.9 \\
\hline $15-19$ & 15,243 & 93.8 & 61.2 & 2,413 & 72.9 & 9.7 & $893^{\S}$ & $74.1^{\S}$ & $3.6^{\S}$ & 6,348 & 25.5 & 24,897 & 119.9 \\
\hline $20-24$ & 11,676 & 71.4 & 56.5 & 2,272 & 72.9 & 11.0 & $841^{\S}$ & $65.1^{\S}$ & $4.1^{\S}$ & 5,894 & 28.5 & 20,683 & 99.7 \\
\hline $25-34$ & 15,981 & 50.9 & 55.2 & 4,044 & 73.3 & 14.0 & $682^{\S}$ & $23.2^{\S}$ & $2.4^{\S}$ & 8,247 & 28.5 & 28,954 & 72.6 \\
\hline $35-44$ & 18,916 & 53.1 & 58.5 & 4,456 & 77.8 & 13.8 & $953^{\S}$ & $34.7^{\S}$ & $3.0^{\S}$ & 7,985 & 24.7 & 32,310 & 73.3 \\
\hline $45-54$ & 16,648 & 48.3 & 57.3 & 4,369 & 88.8 & 15.0 & $479^{\S}$ & $21.4^{\S}$ & $1.6^{\S}$ & 7,572 & 26.0 & 29,068 & 69.9 \\
\hline $55-64$ & 14,064 & 56.7 & 62.2 & 1,829 & 62.8 & 8.1 & $1,165^{\S}$ & $84.7^{\S}$ & $5.2^{\S}$ & 5,543 & 24.5 & 22,601 & 77.7 \\
\hline $65-74$ & 13,455 & 84.0 & 64.1 & 1,679 & 96.5 & 8.0 & $899^{\S}$ & $115.7^{\S}$ & $4.3^{\S}$ & 4,957 & 23.6 & 20,990 & 113.3 \\
\hline$\geq 75$ & 42,735 & 268.0 & 70.6 & 2,644 & 195.5 & 4.4 & $1,370^{\dagger}$ & $255.5^{\dagger}$ & $2.3^{\dagger}$ & 13,761 & 22.7 & 60,510 & 339.3 \\
\hline Total & 169,769 & 71.3 & 61.7 & 28,576 & 74.1 & 10.4 & 8,351 & 50.1 & 3.0 & 68,454 & 24.9 & 275,150 & 93.8 \\
\hline Adjusted ? & & 69.8 & & & 78.7 & & & 58.2 & & & & & 93.6 \\
\hline
\end{tabular}

- Average annual rate per 100,000 population.

† Sample size is 30-59; the value of the estimate was reported but may not be stable.

$\S$ Sample size is less than 30 ; the value of the estimate was also reported, but it is not considered stable.

g) Age-adjusted to the 2000 U.S. standard population.

Note: In-hospital deaths and patients who transferred from another hospital were excluded.

Numbers subject to rounding error. 
TABLE 11: Estimated Average Annual Numbers, Rates, and Percentages of Traumatic Brain Injury-

Related Hospitalizations, by Age Group and External Cause, United States, 2002-2006

\begin{tabular}{|c|c|c|c|c|c|c|c|c|c|c|c|c|c|c|c|c|c|}
\hline \multirow[b]{2}{*}{ AGE (YRS) } & \multicolumn{3}{|c|}{$\begin{array}{l}\text { MOTOR VEHICLE- } \\
\text { TRAFFIC* }\end{array}$} & \multicolumn{3}{|c|}{ FALLS } & \multicolumn{3}{|c|}{ ASSAULT } & \multicolumn{3}{|c|}{ STRUCK BY/AGAINST } & \multicolumn{3}{|c|}{ OTHER/UNKNOWN } & \multicolumn{2}{|c|}{ TOTAL } \\
\hline & NUMBER & RATE' & ROW \% & NUMBER & RATE' & ROW \% & NUMBER & RATE & ROW \% & NUMBER & RATE & ROW \% & NUMBER & RATE & ROW \% & NUMBER & RATE \\
\hline $0-4$ & 2,182 & $\overline{10.9}$ & 14.3 & 6,458 & 32.2 & 42.4 & $893^{\dagger}$ & $4.5^{\dagger}$ & $5.9^{\dagger}$ & $867^{\dagger}$ & $4.3^{\dagger}$ & $5.7^{\dagger}$ & 4,840 & 24.2 & 31.8 & 15,240 & $\overline{76.1}$ \\
\hline $5-9$ & 2,566 & 13.0 & 29.2 & 1,733 & 8.8 & 19.7 & $10^{\S}$ & $0.1^{\S}$ & $0.1^{\S}$ & $543^{\dagger}$ & $2.8^{\dagger}$ & $6.2^{\dagger}$ & 3,946 & 20.0 & 44.8 & 8,798 & 44.7 \\
\hline $10-14$ & 2,111 & 10.1 & 19.0 & 1,892 & 9.0 & 17.0 & $520^{\S}$ & $2.5^{\S}$ & $4.7^{\S}$ & $1,244^{\dagger}$ & $5.9^{\dagger}$ & $11.2^{\dagger}$ & 5,331 & 25.4 & 48.0 & 11,098 & 52.9 \\
\hline $15-19$ & 9,598 & 46.2 & 38.6 & 2,119 & 10.2 & 8.5 & $1,622^{\dagger}$ & $7.8^{\dagger}$ & $6.5^{\dagger}$ & $1,069^{\dagger}$ & $5.1^{\dagger}$ & $4.3^{\dagger}$ & 10,488 & 50.5 & 42.1 & 24,896 & 119.9 \\
\hline $20-24$ & 7,571 & 36.5 & 36.6 & $1,251^{\dagger}$ & $6.0^{\dagger}$ & $6.1^{\dagger}$ & 1,998 & 9.6 & 9.7 & $237^{\S}$ & $1.1^{\S}$ & $1.1^{\S}$ & 9,626 & 46.4 & 46.5 & 20,683 & 99.7 \\
\hline $25-34$ & 9,208 & 23.1 & 31.8 & 3,391 & 8.5 & 11.7 & 3,496 & 8.8 & 12.1 & $888^{\S}$ & $2.2^{\S}$ & $3.1^{\S}$ & 11,970 & 30.0 & 41.3 & 28,953 & 72.6 \\
\hline $35-44$ & 7,744 & 17.6 & 24.0 & 4,738 & 10.7 & 14.7 & 3,529 & 8.0 & 10.9 & $996^{\dagger}$ & $2.3^{\dagger}$ & $3.1^{\dagger}$ & 15,303 & 34.7 & 47.4 & 32,310 & 73.3 \\
\hline $45-54$ & 6,712 & 16.1 & 23.1 & 5,332 & 12.8 & 18.3 & 1,736 & 4.2 & 6.0 & $459^{\S}$ & $1.1^{\S}$ & $1.6^{\S}$ & 14,829 & 35.6 & 51.0 & 29,068 & 69.9 \\
\hline $55-64$ & 3,217 & 11.1 & 14.2 & 5,559 & 19.1 & 24.6 & $1,134^{\S}$ & $3.9^{\S}$ & $5.0^{\S}$ & $515^{\S}$ & $1.8^{\S}$ & $2.3^{\S}$ & 12,176 & 41.9 & 53.9 & 22,601 & 77.7 \\
\hline $65-74$ & 1,918 & 10.3 & 9.1 & 7,392 & 39.9 & 35.2 & $68^{\S}$ & $0.4^{\S}$ & $0.3^{\S}$ & $467^{\S}$ & $2.5^{\S}$ & $2.2^{\S}$ & 11,145 & 60.1 & 53.1 & 20,990 & 113.3 \\
\hline$\geq 75$ & 4,038 & 22.6 & 6.7 & 22,468 & 126.0 & 37.1 & $334^{\S}$ & $1.9^{\S}$ & $0.6^{\S}$ & $504^{\S}$ & $2.8^{\S}$ & $0.8^{\S}$ & 33,164 & 185.9 & 54.8 & 60,508 & 339.3 \\
\hline Total & 56,865 & 19.4 & 20.7 & 62,333 & 21.2 & 22.7 & 15,340 & 5.2 & 5.6 & 7,789 & 2.7 & 2.8 & 132,818 & 45.3 & 48.3 & 275,145 & 93.8 \\
\hline Adjusted ๆ & & 19.4 & & & 21.2 & & & 5.2 & & & 2.7 & & & 45.1 & & & 93.6 \\
\hline
\end{tabular}

* Motor vehicle-traffic includes the following external cause of injury: occupant, motorcyclist, pedal cyclist, pedestrian, other and unspecified person involved in a motor vehicle-traffic incident.

- Average annual rate per 100,000 population.

† Sample size is 30-59; the value of the estimate was reported but may not be stable.
§ Sample size is less than 30; the value of the estimate was also reported, but it is not considered stable.

g Age-adjusted to the 2000 U.S. standard population.

Note: In-hospital deaths and patients who transferred from another hospital were excluded.

Numbers subject to rounding error. 
TABLE 12: Estimated Average Annual Numbers, Rates, and Percentages of Traumatic Brain InjuryRelated Hospitalizations, by Age Group and Specific Motor Vehicle-Traffic (MVT) External Causes,

\section{United States, 2002-2006}

\begin{tabular}{|c|c|c|c|c|c|c|c|c|c|c|c|c|c|c|c|c|}
\hline \multirow[b]{2}{*}{ AGE (YRS) } & \multicolumn{3}{|c|}{ MVT-OCCUPANT } & \multicolumn{3}{|c|}{ MVT- MOTORCYCLE } & \multicolumn{3}{|c|}{ MVT- PEDAL CYCLE } & \multicolumn{3}{|c|}{ MVT- PEDESTRIAN } & \multicolumn{2}{|c|}{$\begin{array}{c}\text { MVT- OTHER } \\
\text { OR UNSPECIFIED }\end{array}$} & \multicolumn{2}{|l|}{ TOTAL } \\
\hline & NUMBER & RATE & ROW \% & NUMBER & RATE' & ROW \% & NUMBER & RATE' & ROW \% & NUMBER & RATE' & ROW \% & NUMBER & RATE ROW \% & NUMBER & RATE \\
\hline $0-4$ & $1,426^{\dagger}$ & $7.1^{\dagger}$ & $65.3^{\dagger}$ & -- & -- & -- & -- & -- & -- & $744^{\S}$ & $3.7^{\S}$ & $34.1^{\S}$ & $12^{\S}$ & $0.1^{\S} \overline{0.6^{\S}}$ & 2,182 & $\overline{10.9}$ \\
\hline $5-9$ & $1,487^{\dagger}$ & $7.5^{\dagger}$ & $57.9^{\dagger}$ & $100^{\S}$ & $0.5^{\S}$ & $3.9^{\S}$ & $147^{\S}$ & $0.7^{\S}$ & $5.7^{\S}$ & $677^{\dagger}$ & $3.4^{\dagger}$ & $26.4^{\dagger}$ & $156^{\S}$ & $0.8^{\S} \quad 6.1^{\S}$ & 2,567 & 13.0 \\
\hline $10-14$ & $984^{\dagger}$ & $4.7^{\dagger}$ & $46.6^{\dagger}$ & $91^{\S}$ & $0.4^{\S}$ & $4.3^{\S}$ & $360^{\dagger}$ & $1.7^{\dagger}$ & $17.0^{\dagger}$ & $650^{\dagger}$ & $3.1^{\dagger}$ & $30.8^{\dagger}$ & $26^{\S}$ & $0.1^{\S} \quad 1.2^{\S}$ & 2,111 & 10.1 \\
\hline $15-19$ & 6,802 & 32.8 & 70.9 & $1,182^{\S}$ & $5.7^{\S}$ & $12.3^{\S}$ & $243^{\S}$ & $1.2^{\S}$ & $2.5^{\S}$ & $650^{\S}$ & $3.1^{\S}$ & $6.8^{\S}$ & $722^{\S}$ & $3.5^{\S} 7.5^{\S}$ & 9,599 & 46.2 \\
\hline $20-24$ & 5,567 & 26.8 & 73.5 & $614^{\S}$ & $3.0^{\S}$ & $8.1^{\S}$ & $143^{\S}$ & $0.7^{\S}$ & $1.9^{\S}$ & $703^{\S}$ & $3.4^{\S}$ & $9.3^{\S}$ & $543^{\S}$ & $2.6^{\S} 7.2^{\S}$ & 7,570 & 36.5 \\
\hline $25-34$ & 6,048 & 15.2 & 65.7 & $1,428^{\dagger}$ & $3.6^{\dagger}$ & $15.5^{\dagger}$ & $357^{\S}$ & $0.9^{\S}$ & $3.9^{\S}$ & $792^{\dagger}$ & $2.0^{\dagger}$ & $8.6^{\dagger}$ & $583^{\S}$ & $1.5^{\S} 6.3^{\S}$ & 9,208 & 23.1 \\
\hline $35-44$ & 5,216 & 11.8 & 67.4 & $1,299^{\dagger}$ & $2.9^{\dagger}$ & $16.8^{\dagger}$ & $66^{\S}$ & $0.1^{\S}$ & $0.8^{\S}$ & $852^{\S}$ & $1.9^{\S}$ & $11.0^{\S}$ & $311^{\S}$ & $0.7^{\S} \quad 4.0^{\S}$ & 7,744 & 17.6 \\
\hline $45-54$ & 4,263 & 10.2 & 63.5 & $1,114^{\dagger}$ & $2.7^{\dagger}$ & $16.6^{\dagger}$ & $131^{\S}$ & $0.3^{\S}$ & $1.9^{\S}$ & $697^{\S}$ & $1.7^{\S}$ & $10.4^{\S}$ & $506^{\S}$ & $1.2^{\S} 7.5^{\S}$ & 6,711 & 16.1 \\
\hline $55-64$ & 1,872 & 6.4 & 58.2 & $434^{\S}$ & $1.5^{\S}$ & $13.5^{\S}$ & $49 \S$ & $0.2^{\S}$ & $1.5^{\S}$ & $498^{\S}$ & $1.7^{\S}$ & $15.5^{\S}$ & $364^{\S}$ & $1.3^{\S} 11.3^{\S}$ & 3,217 & 11.1 \\
\hline $65-74$ & 1,374 & 7.4 & 71.6 & $88^{\S}$ & $0.5^{\S}$ & $4.6^{\S}$ & $28^{\S}$ & $0.2^{\S}$ & $1.5^{\S}$ & $212^{\S}$ & $1.1^{\S}$ & $11.1^{\S}$ & $215^{\S}$ & $1.2^{\S} 11.2^{\S}$ & 1,917 & 10.3 \\
\hline$\geq 75$ & 3,009 & 16.9 & 74.5 & -- & -- & -- & -- & -- & -- & $741^{\S}$ & $4.2^{\S}$ & $18.3^{\S}$ & $289 \S$ & $1.6^{\S} 7.2^{\S}$ & 4,039 & 22.6 \\
\hline Total & 38,048 & 13.0 & 66.9 & 6,350 & 2.2 & 11.2 & 1,524 & 0.5 & 2.7 & 7,216 & 2.5 & 12.7 & 3,727 & $1.3 \quad 6.6$ & 56,865 & 19.4 \\
\hline Adjusted ? & & 12.9 & & & 2.2 & & & 0.5 & & & 2.5 & & & 1.3 & & 19.4 \\
\hline
\end{tabular}

- Average annual rate per 100,000 population.

† Sample size is 30-59; the value of the estimate was reported but may not be stable.

§ Sample size is less than 30; the value of the estimate was also reported, but it is not considered stable.

I Age-adjusted to the 2000 U.S. standard population.

-- No data for these cells.

Note: In-hospital deaths and patients who transferred from another hospital were excluded.

Numbers subject to rounding error. 
TABLE 13: Estimated Average Annual Numbers and Percentages of Traumatic Brain Injury-Related Hospitalizations, by Age Group and Expected Source of Payment, United States, 2002-2006

\begin{tabular}{|c|c|c|c|c|c|c|c|c|c|c|c|}
\hline \multirow[b]{2}{*}{ AGE(YRS) } & \multicolumn{2}{|c|}{ PRIVATE } & \multicolumn{2}{|c|}{ MEDICAID } & \multicolumn{2}{|c|}{ MEDICARE } & \multicolumn{2}{|c|}{ WORKER'S COMPENSATION } & \multicolumn{2}{|c|}{ OTHER/UNKNOWN* } & \multirow{2}{*}{$\begin{array}{c}\text { TOTAL } \\
\text { NUMBER }\end{array}$} \\
\hline & NUMBER & ROW \% & NUMBER & ROW \% & NUMBER & ROW \% & NUMBER & ROW \% & NUMBER & ROW \% & \\
\hline $0-4$ & 7,029 & 46.1 & 6,339 & 41.6 & $54^{\S}$ & $0.4^{\S}$ & -- & -- & 1,818 & 11.9 & 15,240 \\
\hline $5-9$ & 4,823 & 54.8 & 2,659 & 30.2 & -- & -- & -- & -- & 1,317 & 15.0 & 8,799 \\
\hline $10-14$ & 6,690 & 60.3 & 3,031 & 27.3 & -- & -- & -- & -- & 1,376 & 12.4 & 11,097 \\
\hline $15-19$ & 14,619 & 58.7 & 4,863 & 19.5 & $193^{\S}$ & $0.8^{\S}$ & $331^{\S}$ & $1.3^{\S}$ & 4,891 & 19.6 & 24,897 \\
\hline $20-24$ & 9,097 & 44.0 & 3,319 & 16.0 & $407^{\S}$ & $2.0^{\S}$ & $861^{\dagger}$ & $4.2^{\dagger}$ & 6,998 & 33.8 & 20,682 \\
\hline $25-34$ & 12,715 & 43.9 & 4,479 & 15.5 & $848^{\S}$ & $2.9^{\S}$ & 1,337 & 4.6 & 9,574 & 33.1 & 28,953 \\
\hline $35-44$ & 13,062 & 40.4 & 4,051 & 12.5 & 2,495 & 7.7 & 1,899 & 5.9 & 10,804 & 33.4 & 32,311 \\
\hline $45-54$ & 13,743 & 47.3 & 3,516 & 12.1 & 2,702 & 9.3 & $1,648^{\dagger}$ & $5.7^{\dagger}$ & 7,459 & 25.7 & 29,068 \\
\hline $55-64$ & 11,306 & 50.0 & 2,795 & 12.4 & 3,893 & 17.2 & $1,188^{\dagger}$ & $5.3^{\dagger}$ & 3,419 & 15.1 & 22,601 \\
\hline $65-74$ & 3,847 & 18.3 & $728^{\dagger}$ & $3.5^{\dagger}$ & 14,735 & 70.2 & $400^{\S}$ & $1.9^{\S}$ & $1,279^{\dagger}$ & $6.1^{\dagger}$ & 20,989 \\
\hline$\geq 75$ & 7,045 & 11.6 & $500^{\S}$ & $0.8^{\S}$ & 51,278 & 84.7 & $23^{\S}$ & $0.0^{\S}$ & 1,663 & 2.7 & 60,509 \\
\hline Total & 103,976 & 37.8 & 36,280 & 13.2 & 76,605 & 27.8 & 7,687 & 2.8 & 50,598 & 18.4 & 275,147 \\
\hline
\end{tabular}

* Includes self pay, no charge, other government, other, and unknown.

+ Sample size is 30-59; the value of the estimate was reported but may not be stable.

$\S$ Sample size is less than 30 ; the value of the estimate was also reported, but it is not considered stable.

-- No data for these cells.

Note: In-hospital deaths and patients who transferred from another hospital were excluded.

Numbers subject to rounding error. 
TABLE 14: Average Annual Numbers, Rates, and Percentages of Traumatic Brain Injury-Related Deaths, by Age Group and Sex, United States, 2002-2006

\begin{tabular}{|c|c|c|c|c|c|c|c|c|}
\hline \multirow[b]{2}{*}{ AGE (YRS) } & \multicolumn{3}{|c|}{ MALE } & \multicolumn{3}{|c|}{ FEMALE } & \multicolumn{2}{|c|}{ TOTAL* } \\
\hline & NUMBER & RATE & ROW \% & NUMBER & RATE & ROW \% & NUMBER & RATE" \\
\hline $0-4$ & 574 & 5.6 & 57.5 & 424 & 4.3 & 42.5 & 998 & 5.0 \\
\hline $5-9$ & 259 & 2.6 & 57.6 & 191 & 2.0 & 42.4 & 450 & 2.3 \\
\hline $10-14$ & 477 & 4.4 & 65.7 & 249 & 2.4 & 34.3 & 726 & 3.5 \\
\hline $15-19$ & 2,977 & 27.9 & 74.5 & 1,018 & 10.1 & 25.5 & 3,995 & 19.2 \\
\hline $20-24$ & 4,140 & 38.8 & 82.0 & 908 & 9.0 & 18.0 & 5,048 & 24.3 \\
\hline $25-34$ & 5,551 & 27.4 & 81.3 & 1,275 & 6.5 & 18.7 & 6,826 & 17.1 \\
\hline $35-44$ & 5,428 & 24.7 & 77.6 & 1,567 & 7.1 & 22.4 & 6,995 & 15.9 \\
\hline $45-54$ & 5,592 & 27.4 & 78.5 & 1,533 & 7.2 & 21.5 & 7,125 & 17.1 \\
\hline $55-64$ & 3,913 & 28.0 & 77.8 & 1,115 & 7.4 & 22.2 & 5,028 & 17.3 \\
\hline $65-74$ & 3,125 & 36.9 & 73.5 & 1,128 & 11.2 & 26.5 & 4,253 & 22.9 \\
\hline$\geq 75$ & 5,935 & 88.3 & 58.8 & 4,160 & 37.4 & 41.2 & 10,095 & 56.6 \\
\hline Total & 37,971 & 26.3 & 73.7 & 13,568 & 9.1 & 26.3 & 51,539 & 17.6 \\
\hline Adjusted ๆ & & 27.1 & & & 8.6 & & & 17.4 \\
\hline
\end{tabular}

* 128 mortality records (2002-2006) were omitted because of missing age information.

- Average annual rate per 100,000 population.

I Age-adjusted to the 2000 U.S. standard population.

Numbers subject to rounding error. 
TABLE 15: Average Annual Numbers, Rates, and Percentages of Traumatic Brain Injury-Related Deaths, by Age Group and Race, United States, 2002-2006

\begin{tabular}{|c|c|c|c|c|c|c|c|c|c|c|c|}
\hline \multirow[b]{2}{*}{ AGE (YRS) } & \multicolumn{3}{|c|}{ WHITE } & \multicolumn{3}{|c|}{ BLACK } & \multicolumn{3}{|c|}{$\begin{array}{l}\text { AMERICAN INDIAN, ALASKA NATIVE, } \\
\text { ASIAN, OR PACIFIC ISLANDER }\end{array}$} & \multicolumn{2}{|c|}{ TOTAL* } \\
\hline & NUMBER & RATE & ROW \% & NUMBER & RATE" & ROW \% & NUMBER & RATE & ROW \% & NUMBER & RATE \\
\hline $0-4$ & 693 & 4.4 & 69.4 & 253 & 7.8 & 25.3 & 52 & 4.4 & 5.2 & 998 & 5.0 \\
\hline $5-9$ & 336 & 2.2 & 74.6 & 90 & 2.8 & 20.1 & 24 & 2.0 & 5.3 & 450 & 2.3 \\
\hline $10-14$ & 568 & 3.5 & 78.2 & 129 & 3.7 & 17.8 & 29 & 2.4 & 4.0 & 726 & 3.5 \\
\hline $15-19$ & 3,179 & 19.6 & 79.6 & 653 & 19.7 & 16.4 & 163 & 13.5 & 4.1 & 3,995 & 19.2 \\
\hline $20-24$ & 3,841 & 23.5 & 76.1 & 1,000 & 32.1 & 19.8 & 207 & 16.0 & 4.1 & 5,048 & 24.3 \\
\hline $25-34$ & 5,202 & 16.6 & 76.2 & 1,366 & 24.7 & 20.0 & 259 & 8.8 & 3.8 & 6,827 & 17.1 \\
\hline $35-44$ & 5,795 & 16.3 & 82.8 & 976 & 17.0 & 13.9 & 224 & 8.2 & 3.2 & 6,995 & 15.9 \\
\hline $45-54$ & 6,108 & 17.7 & 85.7 & 813 & 16.5 & 11.4 & 204 & 9.1 & 2.9 & 7,125 & 17.1 \\
\hline $55-64$ & 4,440 & 17.9 & 88.3 & 444 & 15.3 & 8.8 & 144 & 10.5 & 2.9 & 5,028 & 17.3 \\
\hline $65-74$ & 3,830 & 23.9 & 90.1 & 301 & 17.3 & 7.1 & 122 & 15.7 & 2.9 & 4,253 & 22.9 \\
\hline$\geq 75$ & 9,409 & 59.0 & 93.2 & 431 & 31.9 & 4.3 & 255 & 47.5 & 2.5 & 10,095 & 56.6 \\
\hline Total & 43,401 & 18.2 & 84.2 & 6,456 & 16.7 & 12.5 & 1,683 & 10.1 & 3.3 & 51,540 & 17.6 \\
\hline Adjusted ๆ & & 17.7 & & & 17.3 & & & 11.2 & & & 17.4 \\
\hline
\end{tabular}

* 128 mortality records (2002-2006) were omitted because of missing age information.

- Average annual rate per 100,000 population.

g) Age-adjusted to the 2000 U.S. standard population.

Numbers subject to rounding error. 
TABLE 16: Average Annual Numbers, Rates, and Percentages of Traumatic Brain Injury-Related Deaths, by Age Group and External Cause, United States, 2002-2006

\begin{tabular}{|c|c|c|c|c|c|c|c|c|c|c|c|c|c|c|c|c|c|}
\hline \multirow[b]{2}{*}{ AGE (YRS) } & \multicolumn{3}{|c|}{$\begin{array}{l}\text { MOTOR VEHICLE- } \\
\text { TRAFFIC* }\end{array}$} & \multicolumn{3}{|c|}{ FALLS } & \multicolumn{3}{|c|}{ ASSAULT } & \multicolumn{3}{|c|}{ STRUCK BY/AGAINST } & \multicolumn{3}{|c|}{ OTHER/UNKNOWN } & \multicolumn{2}{|c|}{ TOTAL $^{+}$} \\
\hline & NUMBER & RATE & ROW \% & NUMBER & RATE' & ROW \% & NUMBER & RATE & ROW \% & NUMBER & RATE' & ROW \% & NUMBER & RATE & ROW \% & NUMBER & RATE \\
\hline $0-4$ & 395 & 2.0 & 39.6 & 37 & 0.2 & 3.7 & 364 & 1.8 & 36.4 & 22 & 0.1 & 2.2 & 180 & 0.9 & 18.1 & 998 & 5.0 \\
\hline $5-9$ & 303 & 1.5 & 67.5 & 10 & 0.0 & 2.1 & 48 & 0.2 & 10.7 & 12 & 0.1 & 2.7 & 76 & 0.4 & 17.0 & 449 & 2.3 \\
\hline $10-14$ & 436 & 2.1 & 60.0 & 15 & 0.1 & 2.0 & 86 & 0.4 & 11.9 & 10 & 0.0 & 1.3 & 180 & 0.9 & 24.8 & 727 & 3.5 \\
\hline $15-19$ & 2,344 & 11.3 & 58.7 & 52 & 0.3 & 1.3 & 634 & 3.1 & 15.9 & 14 & 0.1 & 0.4 & 951 & 4.6 & 23.8 & 3,995 & 19.2 \\
\hline $20-24$ & 2,444 & 11.8 & 48.4 & 95 & 0.5 & 1.9 & 979 & 4.7 & 19.4 & 22 & 0.1 & 0.4 & 1,509 & 7.3 & 29.9 & 5,049 & 24.3 \\
\hline $25-34$ & 2,740 & 6.9 & 40.1 & 182 & 0.5 & 2.7 & 1,315 & 3.3 & 19.3 & 46 & 0.1 & 0.7 & 2,543 & 6.4 & 37.3 & 6,826 & 17.1 \\
\hline $35-44$ & 2,458 & 5.6 & 35.1 & 414 & 0.9 & 5.9 & 982 & 2.2 & 14.0 & 59 & 0.1 & 0.8 & 3,083 & 7.0 & 44.1 & 6,996 & 15.9 \\
\hline $45-54$ & 2,110 & 5.1 & 29.6 & 760 & 1.8 & 10.7 & 712 & 1.7 & 10.0 & 67 & 0.2 & 0.9 & 3,476 & 8.4 & 48.8 & 7,125 & 17.1 \\
\hline $55-64$ & 1,281 & 4.4 & 25.5 & 896 & 3.1 & 17.8 & 349 & 1.2 & 6.9 & 49 & 0.2 & 1.0 & 2,454 & 8.4 & 48.8 & 5,029 & 17.3 \\
\hline $65-74$ & 832 & 4.5 & 19.6 & 1,375 & 7.4 & 32.3 & 169 & 0.9 & 4.0 & 37 & 0.2 & 0.9 & 1,839 & 9.9 & 43.2 & 4,252 & 22.9 \\
\hline$\geq 75$ & 1,053 & 5.9 & 10.4 & 5,882 & 33.0 & 58.3 & 164 & 0.9 & 1.6 & 40 & 0.2 & 0.4 & 2,956 & 16.6 & 29.3 & 10,095 & 56.6 \\
\hline Total & 16,396 & 5.6 & 31.8 & 9,718 & 3.3 & 18.9 & 5,802 & 2.0 & 11.3 & 378 & 0.1 & 0.7 & 19,247 & 6.6 & 37.3 & 51,541 & 17.6 \\
\hline Adjusted ๆ & & 5.6 & & & 3.3 & & & 2.0 & & & 0.1 & & & 6.5 & & & 17.4 \\
\hline
\end{tabular}

* Motor vehicle-traffic includes the following external cause of injury: occupant, motorcyclist, pedal cyclist, pedestrian, other and unspecified person involved in a motor vehicle-traffic incident.

+128 mortality records (2002-2006) were omitted because of missing age information.

- Average annual rate per 100,000 population.

I Age-adjusted to the 2000 U.S. standard population.

Numbers subject to rounding error. 
TABLE 17: Average Annual Numbers, Rates, and Percentages of Traumatic Brain Injury-Related Deaths, by Age Group and Specific Motor Vehicle-Traffic (MVT) External Causes, United States, 2002-2006

\begin{tabular}{|c|c|c|c|c|c|c|c|c|c|c|c|c|c|c|c|c|c|}
\hline \multirow[b]{2}{*}{ AGE (YRS) } & \multicolumn{3}{|c|}{ MVT-OCCUPANT } & \multicolumn{3}{|c|}{ MVT- MOTORCYCLE } & \multicolumn{3}{|c|}{ MVT- PEDAL CYCLE } & \multicolumn{3}{|c|}{ MVT- PEDESTRIAN } & \multicolumn{3}{|c|}{$\begin{array}{c}\text { MVT- OTHER } \\
\text { OR UNSPECIFIED }\end{array}$} & \multicolumn{2}{|c|}{ TOTAL* } \\
\hline & NUMBER & RATE' & ROW \% & NUMBER & RATE' & ROW \% & NUMBER & RATE' & ROW \% & NUMBER & RATE' & ROW \% & NUMBER & RATE & ROW \% & NUMBER & RATE \\
\hline $0-4$ & 174 & 0.9 & 43.9 & 0 & $\ddagger$ & 0.1 & 2 & $\ddagger$ & 0.6 & 97 & 0.5 & 24.6 & 122 & 0.6 & 30.8 & 395 & 2.0 \\
\hline $5-9$ & 131 & 0.7 & 43.2 & 3 & $\ddagger$ & 0.9 & 21 & 0.1 & 7.1 & 60 & 0.3 & 19.6 & 89 & 0.4 & 29.2 & 304 & 1.5 \\
\hline $10-14$ & 200 & 1.0 & 45.9 & 14 & 0.1 & 3.1 & 38 & 0.2 & 8.7 & 76 & 0.4 & 17.3 & 109 & 0.5 & 25.0 & 437 & 2.1 \\
\hline $15-19$ & 1,300 & 6.3 & 55.5 & 87 & 0.4 & 3.7 & 26 & 0.1 & 1.1 & 119 & 0.6 & 5.1 & 812 & 3.9 & 34.7 & 2,344 & 11.3 \\
\hline $20-24$ & 1,245 & 6.0 & 50.9 & 219 & 1.1 & 9.0 & 17 & 0.1 & 0.7 & 132 & 0.6 & 5.4 & 832 & 4.0 & 34.1 & 2,445 & 11.8 \\
\hline $25-34$ & 1,268 & 3.2 & 46.3 & 345 & 0.9 & 12.6 & 34 & 0.1 & 1.2 & 208 & 0.5 & 7.6 & 885 & 2.2 & 32.3 & 2,740 & 6.9 \\
\hline $35-44$ & 1,044 & 2.4 & 42.5 & 373 & 0.8 & 15.2 & 54 & 0.1 & 2.2 & 262 & 0.6 & 10.7 & 726 & 1.6 & 29.5 & 2,459 & 5.6 \\
\hline $45-54$ & 856 & 2.1 & 40.6 & 353 & 0.8 & 16.7 & 56 & 0.1 & 2.7 & 263 & 0.6 & 12.5 & 582 & 1.4 & 27.6 & 2,110 & 5.1 \\
\hline $55-64$ & 530 & 1.8 & 41.4 & 162 & 0.6 & 12.6 & 33 & 0.1 & 2.6 & 181 & 0.6 & 14.2 & 375 & 1.3 & 29.2 & 1,281 & 4.4 \\
\hline $65-74$ & 369 & 2.0 & 44.3 & 45 & 0.2 & 5.5 & 18 & 0.1 & 2.2 & 139 & 0.8 & 16.7 & 260 & 1.4 & 31.3 & 831 & 4.5 \\
\hline$\geq 75$ & 464 & 2.6 & 44.1 & 13 & 0.1 & 1.3 & 13 & 0.1 & 1.3 & 202 & 1.1 & 19.2 & 360 & 2.0 & 34.2 & 1,052 & 5.9 \\
\hline Total & 7,581 & 2.6 & 46.2 & 1,614 & 0.5 & 9.8 & 312 & 0.1 & 1.9 & 1,739 & 0.6 & 10.6 & 5,152 & 1.7 & 31.4 & 16,398 & 5.6 \\
\hline Adjusted ๆ & & 2.6 & & & 0.5 & & & 0.1 & & & 0.6 & & & 1.7 & & & 5.6 \\
\hline
\end{tabular}

* 33 mortality records (2002-2006) were omitted because of missing age information.

- Average annual rate per 100,000 population.

I Age-adjusted to the 2000 U.S. standard population.

¥ Sample size was less than 20 for the 5 years combined, so the rate was suppressed.

Numbers subject to rounding error. 
FIGURE 8: Annual Estimates of All Traumatic Brain Injury-Related Emergency Department Visits, Hospitalizations, and Deaths, United States, 2002-2006

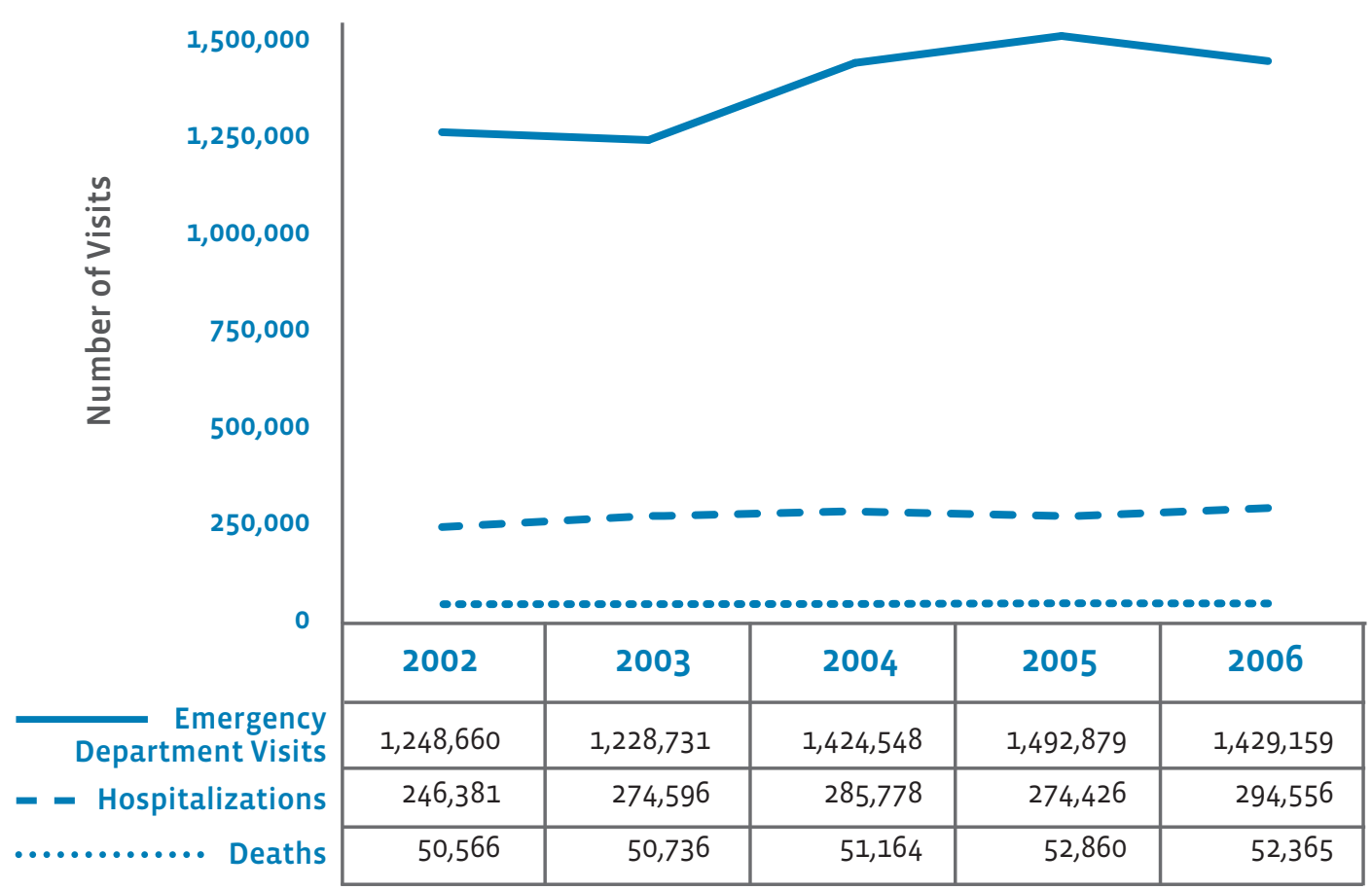

From 2002 to 2006, there was an increase in TBI-related emergency department visits and hospitalizations.

During the period 2002 through 2006, TBI-related emergency department visits increased by $14.4 \%$, hospitalizations increased by $19.5 \%$, and deaths increased by 3.5\%. The estimated population in the United States increased by 3.8\% during the same period. 
FIGURE 9: Annual Rate Estimates of Fall-Related Traumatic Brain Injury-Related Emergency Department Visits, Hospitalizations, and Deaths Among Children Aged 0-14, United States, 2002-2006

From 2002 to 2006, there was an increase in fallrelated TBIs in emergency department visits among children aged 14 years and younger.

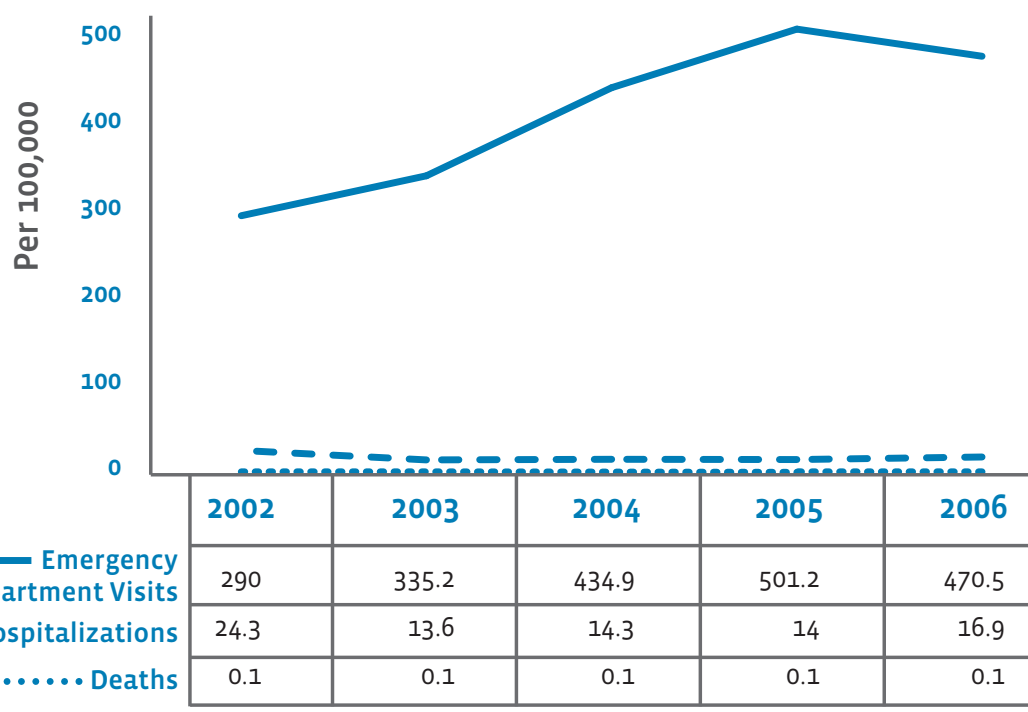

From 2002 to 2006, there was a $62 \%$ increase in fall-related TBIs in emergency department visits among children aged 14 years and younger. Hospitalizations decreased by $30 \%$ and deaths remained the same.
FIGURE 10: Annual Rate Estimates of Fall-Related Traumatic Brain Injury-Related Emergency Department Visits, Hospitalizations, and Deaths Among Adults Aged 65 and older, United States, 2002-2006

From 2002 to 2006, there was an increase in fallrelated TBIs in emergency department visits among adults aged 65 years and older.

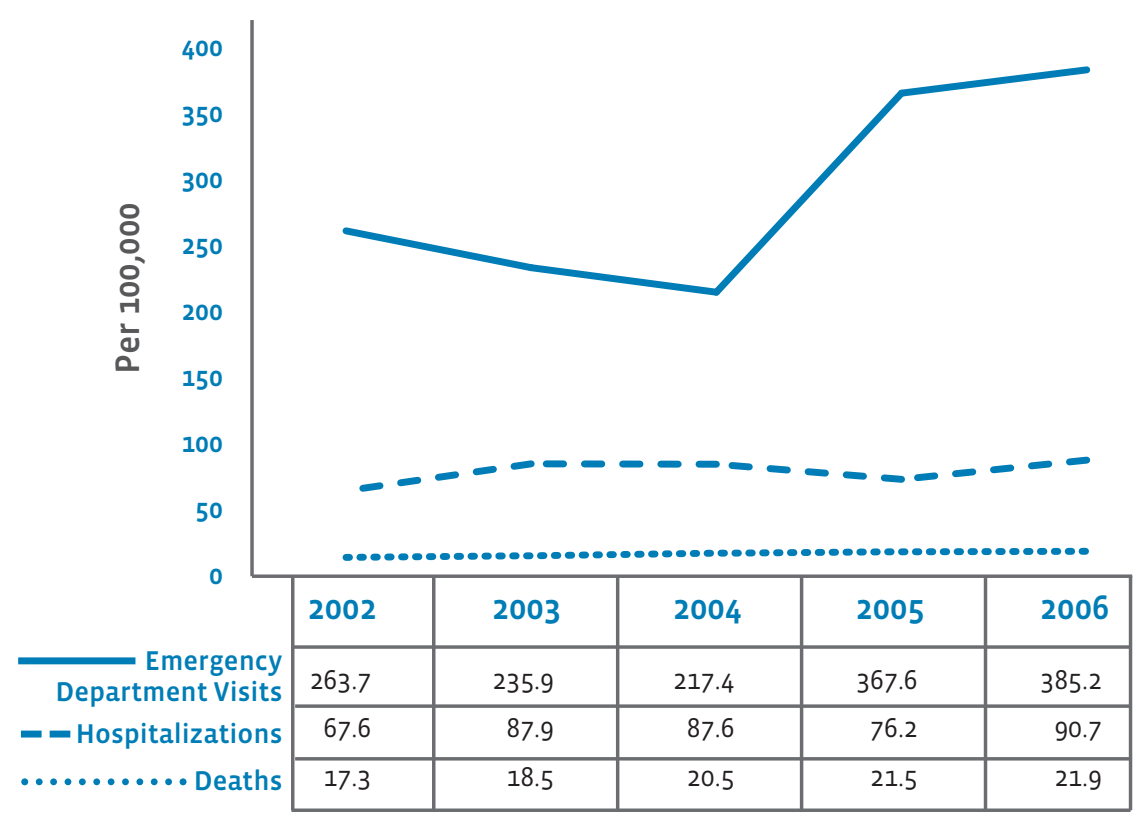

From 2002 to 2006, fall-related TBI rates increased in the older adult population. There were large increases in emergency department visits (46\%), hospitalizations (34\%), and deaths $(27 \%)$ among this age group. 


\title{
Appendix B:
}

\section{Methods and Data Sources}

\author{
Data Sources \\ 49 \\ Identification of TBI Cases \\ 51 \\ External Cause of Injury \\ 55 \\ Population Data \\ 57 \\ Statistical Analysis \\ 59 \\ Limitations \\ 61
}




\section{APPENDIX B: METHODS AND DATA SOURCES}

The data presented in this report were based on three different national data sources: the National Hospital Discharge Survey (NHDS), the National Hospital Ambulatory Medical Care Survey (NHAMCS) and the National Vital Statistics System (NVSS).

These data sources were selected because national estimates for traumatic brain injuries can be calculated using visits to emergency departments, hospitalizations, and deaths. Data for the years of 2002 to 2006 were used to obtain the most recent estimates of the burden of traumatic brain injuries and to increase the stability of the estimated numbers and rates for hospitalizations and emergency department visits. 


\section{DATA SOURCES}

\section{Emergency Department Visits}

The National Hospital Ambulatory Medical Care Survey (NHAMCS), conducted by CDC's National Center for Health Statistics (NCHS), was used to characterize TBIs treated in emergency departments in the United States. The target population of the NHAMCS was in-person visits made in the United States to emergency departments and outpatient departments of nonfederal, short-stay hospitals (hospitals with an average stay of less than 30 days), hospitals that specialize in general medicine or surgical procedures, and children's hospitals. Of the NHAMCS data, only emergency department visits were included in this report. The NHAMCS used a four-stage probability design with the stages being primary sampling units (PSUs), hospitals within PSUs, clinics or emergency departments within hospitals, and patient visits within clinics or emergency departments. Hospital staff were asked to complete patient record forms for a systematic random sample of patient visits occurring during a randomly assigned four-week reporting period. Each visit was assigned a sample weight based on the inverse probability of selection with adjustments for nonresponse. The individual sample weights were summed to produce national estimates of TBI-related emergency department visits. For age, sex, and race, missing values were imputed by randomly assigning a value from a record with similar emergency department volume, geographic region, immediacy with which the patient should be seen, and primary diagnosis. Additional information about the NHAMCS emergency department component is available elsewhere. ${ }^{2}$

For this report, TBI-related cases were selected if one of the three diagnosis fields contained an ICD-9-CM diagnosis code for TBI ${ }^{6}$ (see Table 18). The external cause of injury (E-code) was assigned based on the first E-code field. Emergency department patients who died in the emergency department, who were later hospitalized or transferred to another facility were excluded from the analysis of emergency department visits. During 2002 to 2006, the number of hospitals that participated in the survey ranged from 352 to 406 (more than $91 \%$ of eligible, sampled hospitals each year), with the total number of unweighted emergency department visits ranged from 33,605 to 40,253. The annual number of unweighted TBIrelated emergency department visits identified in the sample ranged from 412 to 460 for a total of 2,198 TBI-related emergency department visits sampled during 2002 to 2006.

\section{Hospitalizations}

The National Hospital Discharge Survey (NHDS) of the NCHS was used to estimate annual number and rates of TBI-related hospitalizations. The NHDS provided data on discharges from nonfederal, short-stay hospitals (those with an average length of stay for all patients of less than 30 days), general (medical or 
surgical) hospitals, or children's general hospitals in the United States. The NHDS used a modified, three-stage probability designed to select records, with the stages being PSUs, hospitals within the PSUs, and discharges within the hospitals. The modification of the design involved selection with certainty of the largest PSUs and hospitals. Demographic and medical data were collected for the selected discharges, and weights were assigned based on the inverse probability of selection with adjustments for non-response. The individual record weights were summed to produce estimates of TBI-related hospitalizations each year for the total United States population. Additional information about the NHDS data is available elsewhere.?

For this report, TBI-related cases were selected if one of the diagnosis fields contained an ICD-9-CM diagnosis code for TBI ${ }^{3}$ (see Table 18). External Cause codes, or E-codes, were contained within the seven diagnosis fields, and the external cause of injury was classified using the first E-code that appeared in the list of codes. Hospitalized patients who died during hospitalization or were transferred to another hospital were excluded from the analysis. During 2002 to 2006, the number of hospitals that provided data for the survey ranged from 426 to 445 (88.9\% to $93.9 \%$ of eligible, sampled hospitals), and the total number of unweighted discharges ranged from 319,530 to 376,328 . The annual number of unweighted TBI-related hospitalizations identified in the sample ranged from 2,104 to 2,583 for a total of 11,880 unweighted TBI-related discharges sampled during 2002 to 2006.

\section{Deaths}

Multiple cause-of-death data from the mortality files of the National Vital Statistics System (NVSS) were used to describe TBI-related deaths. In the United States, state laws require completion of death certificates for all deaths; federal law mandates national collection and publication of deaths and other vital statistics. The NVSS, the federal compilation of these data, is the result of cooperation between NCHS and the states to provide access to statistical information from death certificates. Additional information about these data is available elsewhere. ${ }^{4}$

For this report, TBI-related cases were selected if an ICD-10 diagnosis code ${ }^{5}$ (see Table 19) for TBI appeared in Part I of the death certificate. The E-code was obtained from the underlying cause of death field. 


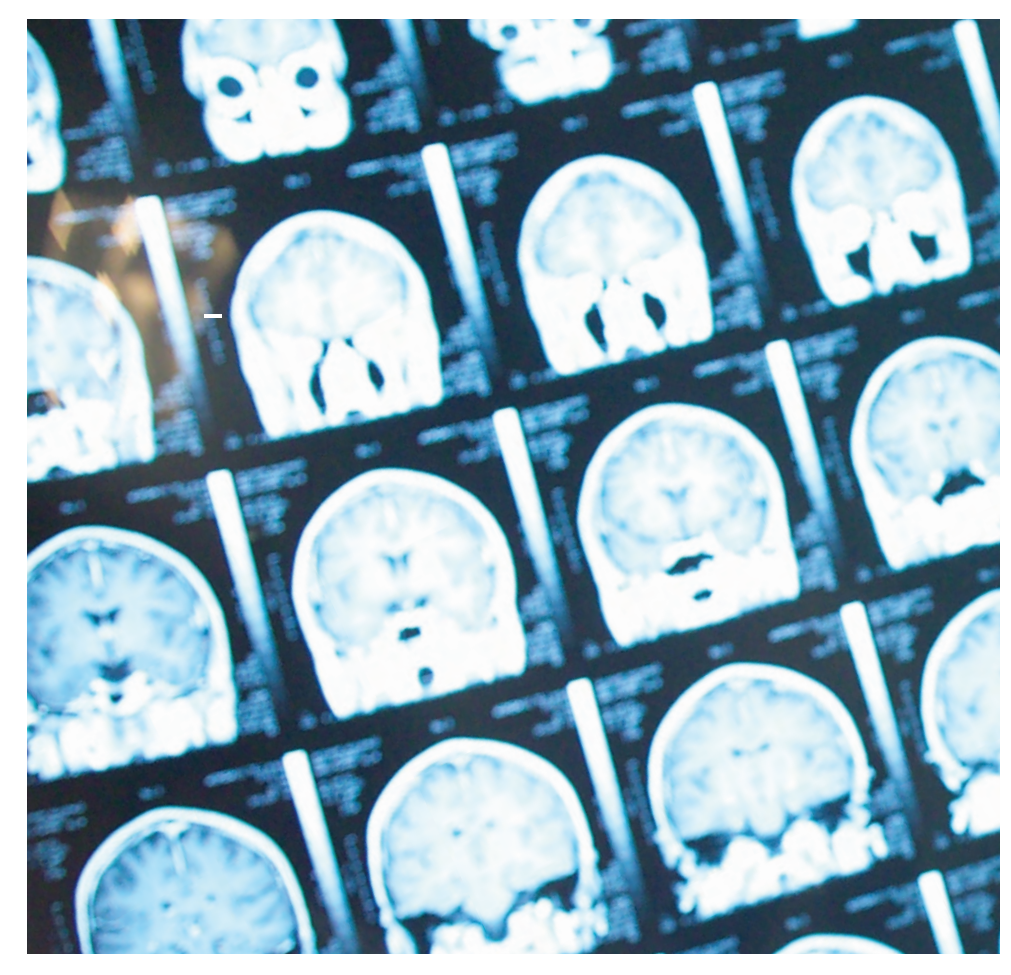
definition. ${ }^{6,7}$ All NHDS and NHAMCS records that contained in one or more of the diagnosis data fields the ICD-9-CM diagnosis codes in the appropriate range, as shown in Table 18, were identified as a TBI emergency department visit or hospitalization. All records that contained in Part I of the death certificate the ICD-10 diagnosis codes (for years 2002-2006) in the appropriate range, as shown in Table 19, were identified as a TBI death.

A record was counted only once regardless of the number of diagnosis codes that met the criteria for TBI. The increased use of $959.01^{5}$ was accompanied by a corresponding drop in the use of 854 . Thus, to avoid underestimating TBIs, cases coded as 959.01 were included. This is consistent with a previous CDC publication on $\mathrm{TBI}^{8}$ and the current $\mathrm{CDC}$ TBI case definition. ${ }^{6,7}$

\section{IDENTIFICATION OF TRAUMATIC BRAIN INJURY CASES}


TABLE 18: ICD-9-CM Codes for Traumatic Brain Injury-Related Emergency Department Visits and Hospitalizations (2002-2006)

\begin{tabular}{lc}
\multicolumn{1}{c}{ DESCRIPTION } & $\begin{array}{c}\text { ICD-9-CM } \\
\text { ICISIS AND HOSPITALIZATIONS) }\end{array}$ \\
\hline Fracture of the vault or base of the skull & $800.0-801.9$ \\
\hline Other and unqualified multiple fractures of the skull & $803.0-804.9$ \\
Intracranial injury, including concussion, contusion, laceration, and hemorrhage & $850.0-854.1$ \\
\hline Injury to optic nerve and pathways & $950.1-950.3$ \\
Shaken baby syndrome & 995.55 \\
\hline Head injury, unspecified & 959.01
\end{tabular}


TABLE 19: ICD-10 Codes for Traumatic Brain Injury-Related Deaths (2002-2006)

\section{DESCRIPTION}

Open wound of the head

Fracture of the skull and facial bones

Injury to optic nerve and pathways

Intracranial injury

Crushing injury of head

Other unspecified injuries of head

Open wounds involving head with neck

Fractures involving head with neck

Crushing injuries involving head with neck

Injuries of brain and cranial nerves with injuries of nerves and spinal cord at neck level

Sequelae of injuries of head
ICD-10 (DEATHS)

S01.0-S01.9

S02.0, S02.1, S02.3, S02.7-S02.9

S04.0

S06.0-S06.9

S07.0, S07.1, S07.8, S07.9

S09.7-S09.9

T01.0

T02.0

T04.0

T06.0

T90.1, T90.2, T90.4, T90.5, T90.8, T90.9 


\section{EXTERNAL CAUSE OF INJURY}

External cause of injury categorization was based on E-codes and classified using categories adapted from CDC's recommended frameworks for presenting injury data..$^{9,10}$ The categories used are presented in Table 20.

Several changes occurred in the classification of external cause of injury between ICD-9 and ICD-10, including the prefixes used to distinguish external cause (from E-codes for ICD-9 to codes beginning with $\mathrm{V}, \mathrm{W}, \mathrm{X}, \mathrm{Y}$, and $* \mathrm{U}$ [terrorism] for ICD-10) and the organization of transport incident codes (based on type of vehicle in ICD-9 and characteristics of the injured person in ICD-10). For this report, the external cause of injury categories were motor vehicle-traffic, unintentional falls, assaults, and struck by/against.

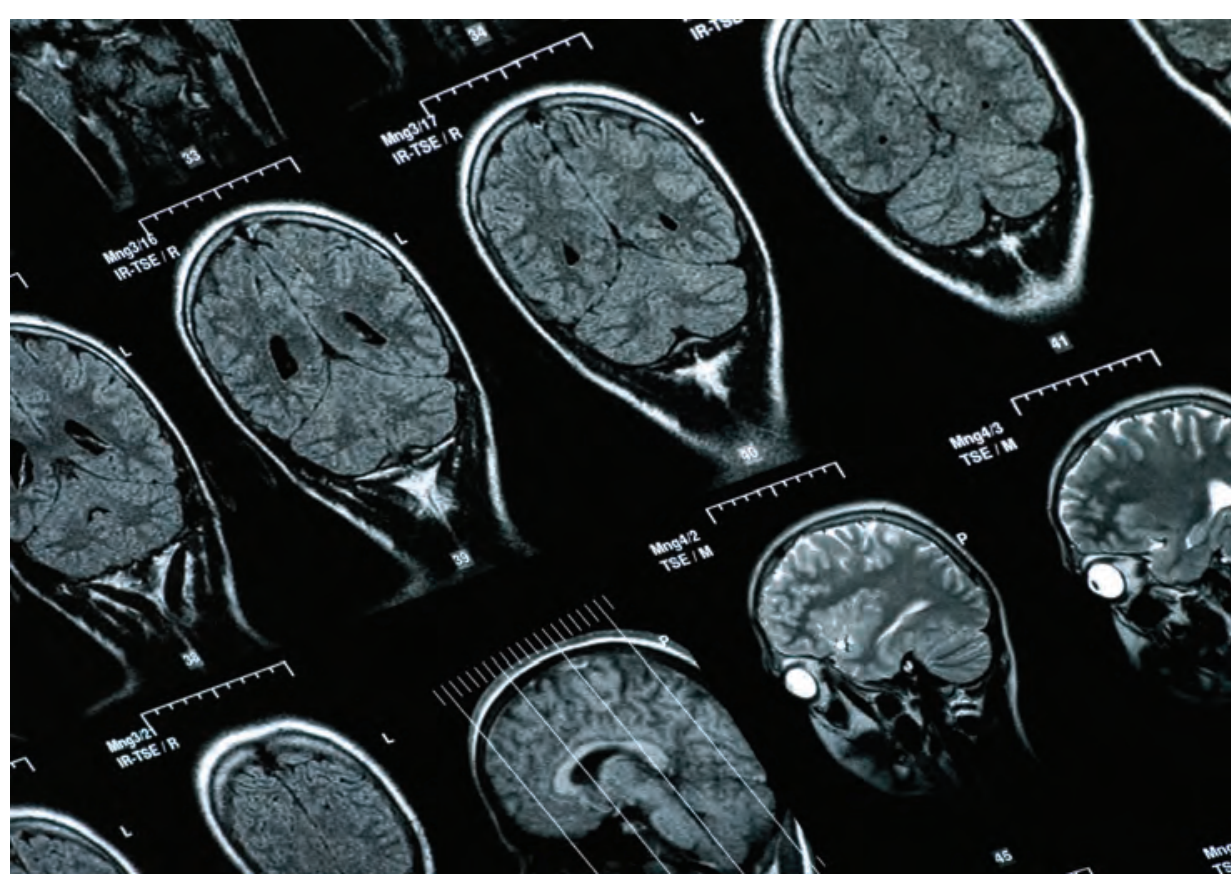

Struck by/against events are those in which a person was struck unintentionally by another person or an object, such as falling debris or objects, struck against an object, such as a wall or another person. For this report, only unintentional and undetermined struck by/ against events were included. Struck by/against events related to assaults were in the assault category. Struck by/against events were reported for all age groups, even though sometimes small sample sizes produced estimates that were not considered stable. 
TABLE 20: External Cause of Injury Categorization for ICD-9-CM Codes (Emergency Department Visits and Hospitalizations, 2002-2006) and ICD-10 Codes (Deaths, 2002-2006)

\section{DESCRIPTION}

Motor vehicle traffic-related (unintentional)

Motorcycle

Pedal cycle

Pedestrian

Other and unspecified

Falls (unintentional and undetermined)

Assault (includes firearm and other)

Struck by and against

Other and unspecified
ICD-9-CM

E810-E819

\section{ICD-10}

V02-V04 (.1, .9), V09.2, V12-V14 (.3-.9), V19 (.4-.6),

V20-V28 (.3-.9), V29 (.4-.9), V30-V79 (.4-.9), V80 (.3-.5),

V81.1, V82.1, V83-V86 (.0-.3), V87 (.0-.8), V89.2

V30-V79 (.4-.9), V81.1, V82.1, V83-V86 (.0-.3)

V20-V28 (.3-.9), V29 (.4-.9)

V12-V14 (.3-.9), V19 (.4-.6)

V02-V04 (.1, .9), V09.2

$\operatorname{E810-E819~(.7)~}$

E810-E819 (.4, .5, .8, .9) V80 (.3-.5), V87 (.0-.8), V89.2

E880-E886, E888, E987 W00-W19, Y30

E960-E969 X85-Y09, Y87.1

E916, E917 W20-W22, W50-W52, Y29

All other E-codes All other cause codes 


\section{POPULATION DATA}

This report uses the United States' Census bridged race population estimates from 2002 to 2006 obtained from NCHS. ${ }^{11}$ The average annual population, derived by dividing the total population by five, is presented in Table 21. The 2000 standard population from the U.S. Bureau of the Census was used to calculate the age-adjusted rates by using the direct method. ${ }^{12}$ The weights applied to the average annual population from the 2000 standard population are also presented in Table 21.

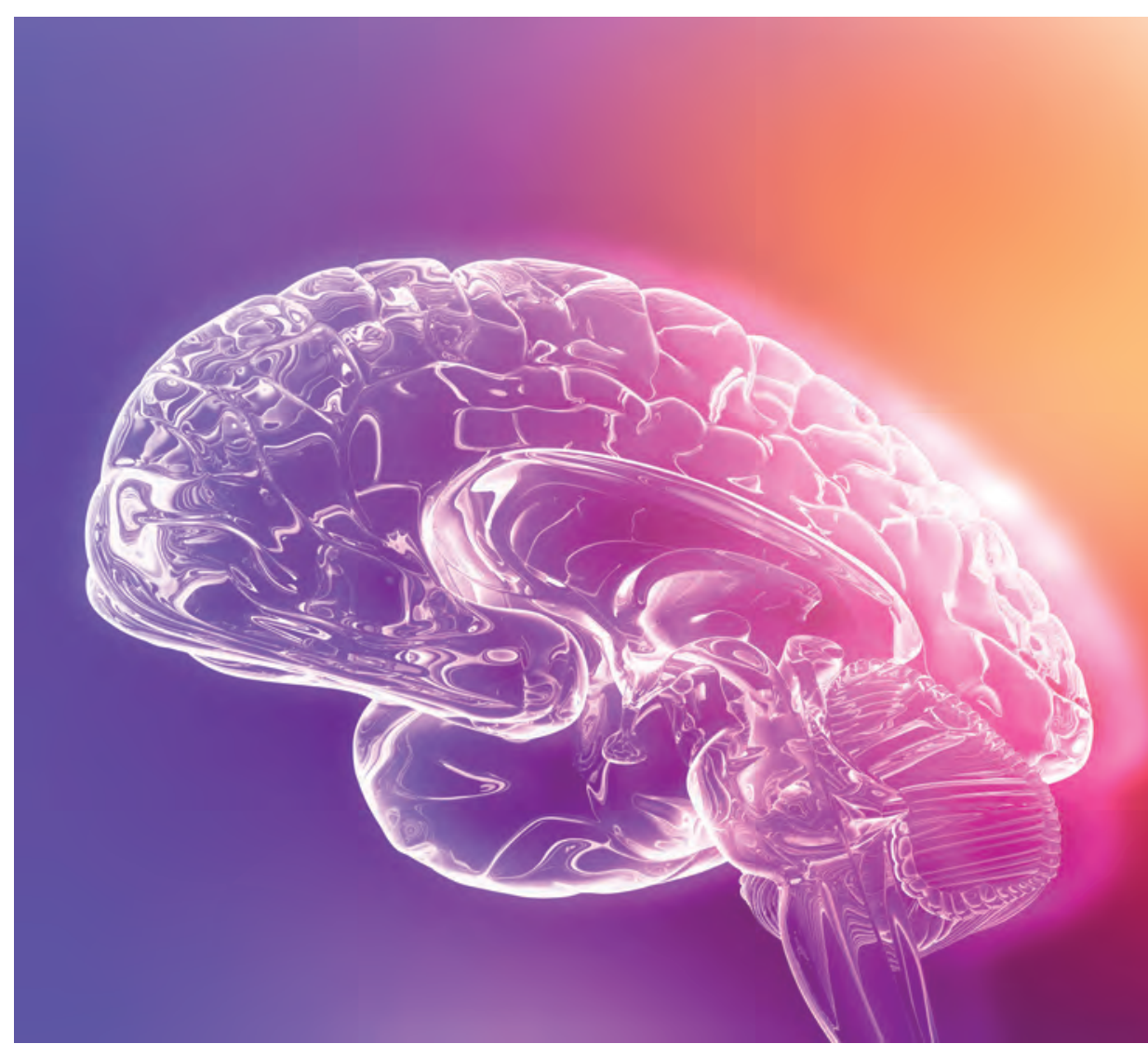


TABLE 21: Estimated Average Annual 2002-2006 Population by Age Group, Sex, and Race; Weights for 2000 Standard Population by Age Group

\begin{tabular}{|c|c|c|c|c|c|c|c|}
\hline \multirow[b]{2}{*}{ AGE (YRS) } & \multicolumn{2}{|c|}{ SEX } & \multicolumn{3}{|c|}{ RACE } & \multirow[b]{2}{*}{ TOTAL } & \multirow[b]{2}{*}{ WEIGHTS* } \\
\hline & MALE & FEMALE & WHITE & BLACK & $\begin{array}{l}\text { AMERICAN INDIAN, } \\
\text { ALASKA NATIVE, ASIAN, } \\
\text { PACIFIC ISLANDER }\end{array}$ & & \\
\hline $0-4$ & $10,240,416$ & $9,784,061$ & $15,582,248$ & $3,260,109$ & $1,182,120$ & $20,024,477$ & 0.0691356496 \\
\hline $5-9$ & $10,081,063$ & $9,624,631$ & $15,332,058$ & $3,200,397$ & $1,173,239$ & $19,705,694$ & 0.0725328983 \\
\hline $10-14$ & $10,740,169$ & $10,229,818$ & $16,259,412$ & $3,503,737$ & $1,206,837$ & $20,969,987$ & 0.0730317441 \\
\hline $15-19$ & $10,658,609$ & $10,106,668$ & $16,251,474$ & $3,309,403$ & $1,204,400$ & $20,765,277$ & 0.0721687774 \\
\hline $20-24$ & $10,671,421$ & $10,077,694$ & $16,341,821$ & $3,115,714$ & $1,291,580$ & $20,749,115$ & 0.0664775665 \\
\hline $25-34$ & $20,226,113$ & $19,659,025$ & $31,421,296$ & $5,519,693$ & $2,944,149$ & $39,885,138$ & 0.1355731628 \\
\hline $35-44$ & $22,007,839$ & $22,085,520$ & $35,614,403$ & $5,731,205$ & $2,747,751$ & $44,093,359$ & 0.1626127865 \\
\hline $45-54$ & $20,443,508$ & $21,159,542$ & $34,445,963$ & $4,920,675$ & $2,236,412$ & $41,603,050$ & 0.1348339972 \\
\hline $55-64$ & $13,997,124$ & $15,075,707$ & $24,786,789$ & 2,911,295 & $1,374,746$ & $29,072,831$ & 0.0872470269 \\
\hline $65-74$ & $8,458,345$ & $10,072,055$ & $16,013,437$ & $1,740,204$ & 776,758 & $18,530,399$ & 0.0660369801 \\
\hline$\geq 75$ & $6,724,189$ & $11,112,062$ & $15,947,766$ & $1,352,384$ & 536,101 & $17,836,250$ & 0.0603494104 \\
\hline Total & $144,248,796$ & $148,986,783$ & $237,996,667$ & $38,564,816$ & $16,674,093$ & $293,235,577$ & \\
\hline
\end{tabular}

* Based on the 2000 standard population.

Numbers subject to rounding error. 


\section{STATISTICAL ANALYSIS}

SAS software ${ }^{13}$ was used to calculate average annual numbers, rates, row percentages, age-adjusted rates, and total numbers.

Average annual numbers were calculated by adding the numbers for all five years and dividing the totals by 5 . Average annual rates were calculated by dividing the total number for all five years by the total population for all five years. Row percentages were calculated by dividing each number by the total number for all five years. Because numbers, rates, and row percentages were all calculated before rounding and were based on the totals for all five years and not the annual average, some results may not be consistent across tables.

An age adjustment rate was made using the direct method to eliminate differences in observed rates that result from age differences in the population distribution. This adjustment was done to allow more accurate comparisons of two or more populations at one point in time or a single population at two or more points in time.

Age-adjusted rates were calculated by the direct method as follows:

$$
\sum_{i=1}^{n}\left(r_{i} \times w_{i}\right)
$$

Where $\boldsymbol{r}_{\boldsymbol{i}}=$ age-specific rates for the population of interest,

$\boldsymbol{W}_{\boldsymbol{i}}=$ age-specific weight based on the 2000 U.S. standard population, and

$\boldsymbol{n}=$ total number of age groups over the age range of the age-adjusted rate.
Age adjustment by the direct method requires use of a standard age distribution; in this report, the year 2000 standard population was selected (see Table 21).

Based on the complex sample design of the NHDS and the NHAMCS, estimates of the number and rate of TBIs requiring emergency department treatment or hospitalization were reported based on the NCHS guidelines below: ${ }^{2}$

- If the sample size was less than 30 , the value of the estimates was reported, but it was not considered stable.

- If the sample size was 30 to 59, the value of the estimate was reported, but it may not be stable.

For the death data, if the sample size was less than 20 for the 5 years combined, the rates were suppressed because the data were not considered stable. ${ }^{12}$ 


\section{LIMITATIONS}

- Three different data sources were used. Results should be interpreted with caution because differences in study methods may have influenced the findings. The NHDS and NHAMCS were based on a sample of inpatients who were discharged from nonfederal short-stay hospitals (NHDS) and emergency department visits (NHAMCS), while multiple cause-of-death data (NVSS) included all deaths.

- The potential for sampling bias exists with any survey. NHDS and NHAMCS procedures assure this possibility is reduced by using stratified sampling of hospitals, random selection of discharges within hospitals and visits within emergency departments, and even distribution of sampling throughout the year.

- The overall burden of TBI in the United States was underestimated. An estimated 439,000 TBIs treated by physicians during office visits and 89,000 treated in outpatient settings were not included in this report. ${ }^{14}$ In addition, TBIs with no medical advice sought, an estimated $25 \%$ of all mild and moderate TBIs, were not included. ${ }^{15}$

- This report does not include TBIs from federal, military, or Veterans Administration (VA) hospitals.

- The lack of external cause of injury coding (E-coding) was potentially problematic. For the NHDS data, one third of cases were missing an E-code. Increased E-code reporting could increase the rates by external cause. Only data by external cause for the leading causes of TBI (falls, struck by/against, motorvehicle/traffic and assault) for the three data sets combined were reported. The actual leading causes varied among emergency department visits, hospitalizations, and deaths. Causes beyond those were combined as other/unknown due to limitations in sample size and consistency among all three data sources. As a result, some causes were not included individually, such as firearm injuries, which is a factor in some TBI deaths. ${ }^{16,17}$ These types of injuries were included in the "other" category.

- E-codes may not capture all of the injuries attributable to a particular cause or intent, especially controversial ones such as assault. Among children, $25 \%$ of all injuries resulting from assaults may not be accounted for by E-codes. ${ }^{18}$ Assaults may not be clinically recognized, especially in vulnerable populations, such as children ${ }^{19}$ and older adults. ${ }^{20}$

- Injury severity was not included in this report for two reasons. First, a measure of severity could not be uniformly applied to all three data sets. ICDMAP-90, ${ }^{21}$ a computer algorithm that converts ICD-9-CM diagnosis codes to a 6-level score approximating the Abbreviated Injury Scale (AIS), was only applicable to NHDS and NHAMCS data. Second, the ICDMAP- 90 has not been updated to include the ICD code 959.01, which is now part of the TBI ICD coded definition. 
FIGURE 11: Sources of Potential Case Duplication When Combining Traumatic Brain Injury-Related Emergency Department Visits, Hospitalizations, and Deaths

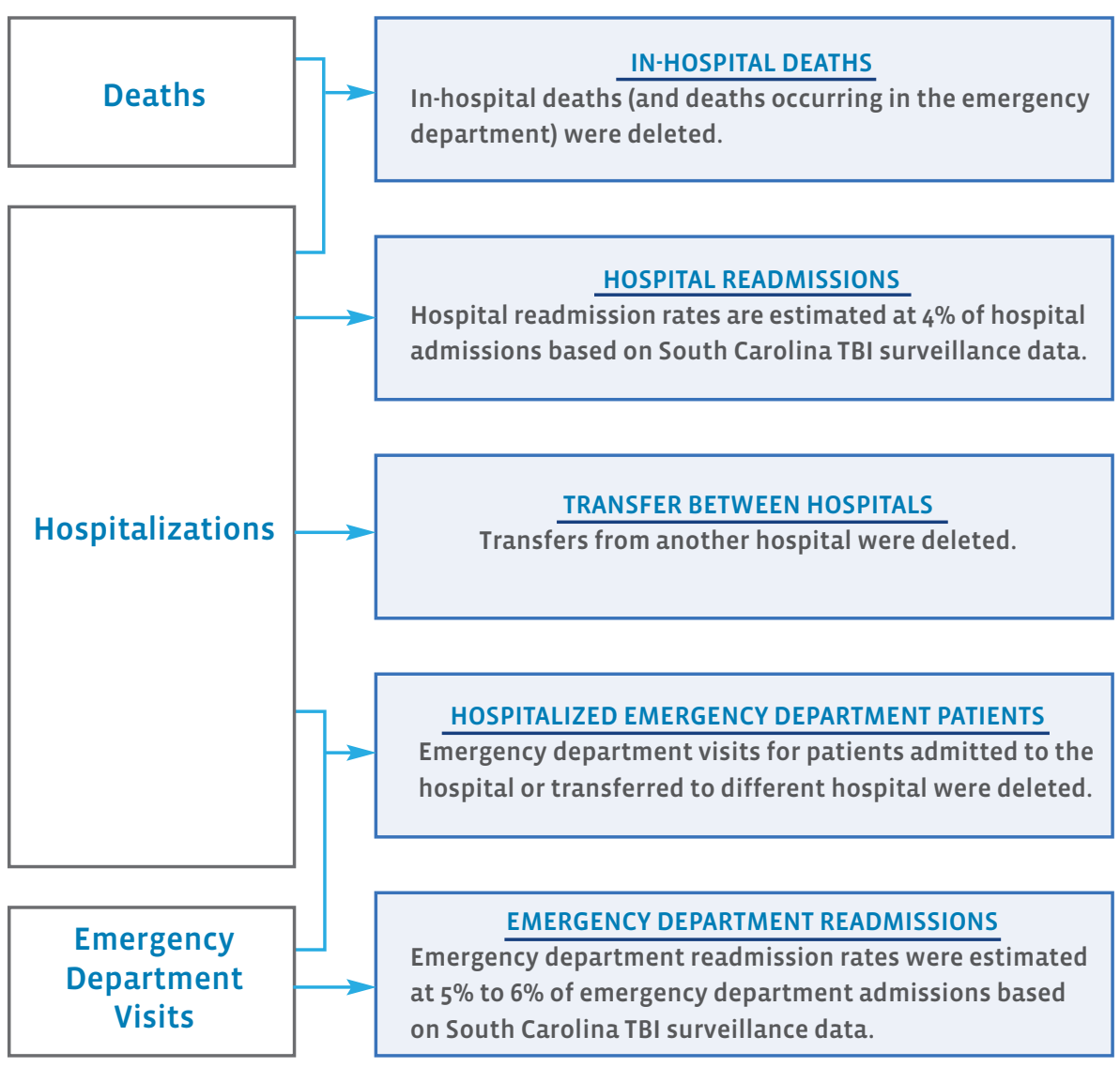

- The NHDS and NHAMCS data are based on hospitalizations and visits to emergency departments - not on individual persons. It is not possible to unduplicate cases in which individuals were hospitalized or treated in emergency departments more than once for the same injury. This limitation precludes calculating the true incidence of $\mathrm{TBI}$; however, the effects on the data are assumed to be quite small. Specifically, data from a population-based follow-up study in South Carolina indicated a readmission rate to the hospital of approximately $4 \%$ and a readmission rate to the emergency department of $5 \%$ to $6 \% .{ }^{22}$ Patients who transferred from another hospital were excluded in the hospitalization tables. Patients who have been hospitalized and later died from their injuries could be another source of over-counting. However, deaths that occurred among patients hospitalized or treated at emergency departments were excluded based on information available in the hospital discharge and emergency department data sets. Patients who were seen in the emergency department and later hospitalized could also be double counted; however, documented hospitalizations were excluded from those counted as seen in the emergency department. Emergency department patients reported to have been transferred were also excluded to help limit double counting. Because none of these data sets are mutually exclusive, the combined number or rate of TBI might be overestimated because some cases could still be double counted (see Figure 11). 


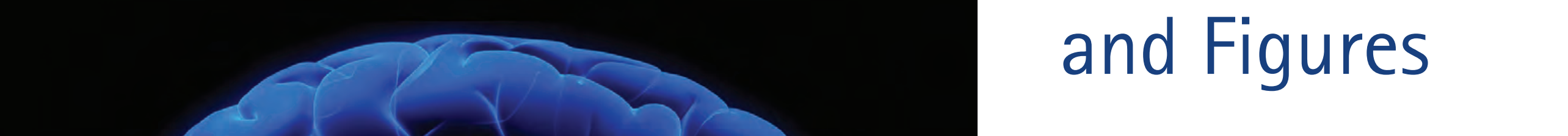

Index of Tables and Figures 


\section{INDEX OF TABLES AND FIGURES}

Figure 1: Estimated Average Annual Number of Traumatic Brain Injury-Related Emergency Department Visits,

Hospitalizations, and Deaths, United States, 2002-2006

Table A: Estimated Percentage of All Injuries and Traumatic Brain Injury-Related Emergency Department Visits, Hospitalizations, and Deaths, United States, 2002-2006

Table B: Estimated Average Annual Numbers of Traumatic Brain Injury-Related Emergency Department Visits, Hospitalizations, and Deaths, by Age Group, United States, 2002-2006

Figure 2: Estimated Average Annual Rates of Traumatic Brain Injury-Related Emergency Department Visits,

Hospitalizations, and Deaths, by Age Group, United States, 2002-2006

Table C: Estimated Average Annual Numbers of Traumatic Brain Injury-Related Emergency Department Visits, Hospitalizations, and Deaths, by Sex, United States, 2002-2006

Figure 3: Estimated Average Annual Rates of Traumatic Brain Injury-Combined Emergency Department Visits, Hospitalizations, and Deaths, by Sex, United States, 2002-2006

Table D: Estimated Average Annual Numbers of Traumatic Brain Injury-Related Emergency Department Visits, Hospitalizations, and Deaths, by External Cause, United States, 2002-2006

Figure 4: Estimated Average Annual Rates of Traumatic Brain Injury-Combined Emergency Department Visits, Hospitalizations, and Deaths, by External Cause, United States, 2002-2006

Figure 5: Estimated Average Percentage of Annual Traumatic Brain Injury-Combined Emergency Department Visits, Hospitalizations, and Deaths, by External Cause, United States, 2002-2006

Figure 6: Estimated Average Percentage of Annual Traumatic Brain Injury-Combined Emergency Department Visits, Hospitalizations, and Deaths Among Children 0 to 14 Years, by External Cause, United States, 2002-2006

Figure 7: Estimated Average Percentage of Annual Traumatic Brain Injury-Combined Emergency Department Visits, Hospitalizations, and Deaths Among Adults 65 Years and Older, by External Cause, United States, 2002-2006 
Table 1: Estimated Average Annual Numbers, Rates, and Percentages of Traumatic Brain Injury-Related

Emergency Department Visits, Hospitalizations, and Deaths, by Age Group, United States, $2002-2006$

Table 2: Estimated Average Annual Numbers and Percentages of Traumatic Brain Injury-Related Emergency Department Visits, by Age Group and Disposition, United States, 2002 - 2006

Table 3: Estimated Average Annual Numbers, Rates, and Percentages of Traumatic Brain Injury-Related Emergency Department Visits, by Age Group and Sex, United States, 2002 - 2006

Table 4: Estimated Average Annual Numbers, Rates, and Percentages of Traumatic Brain Injury-Related Emergency Department Visits, by Age Group and Race, United States, 2002 - 2006

Table 5: Estimated Average Annual Numbers, Rates, and Percentages of Traumatic Brain Injury-Related Emergency Department Visits, by Age Group and External Cause, United States, 2002 - 2006

Table 6: Estimated Average Annual Numbers, Rates, and Percentages of Traumatic Brain Injury-Related Emergency Department Visits, by Age Group and Specific Motor Vehicle - Traffic (MVT) External Causes, United States, 2002 - 2006

Table 7: Estimated Average Annual Numbers and Percentages of Traumatic Brain Injury-Related

Emergency Department Visits, by Age Group and Expected Source of Payment, United States, $2002-2006$

Table 8: Estimated Average Annual Numbers and Percentages of Traumatic Brain Injury-Related Hospitalizations, by Age Group and Disposition, United States, $2002-2006$

Table 9: Estimated Average Annual Numbers, Rates, and Percentages of Traumatic Brain Injury-Related Hospitalizations, by Age Group and Sex, United States, $2002-2006$

Table 10: Estimated Average Annual Numbers, Rates, and Percentages of Traumatic Brain Injury-Related Hospitalizations, by Age Group and Race, United States, $2002-2006$

Table 11: Estimated Average Annual Numbers, Rates, and Percentages of Traumatic Brain Injury-Related Hospitalizations, by Age Group and External Cause, United States, $2002-2006$

Table 12: Estimated Average Annual Numbers, Rates, and Percentages of Traumatic Brain Injury-Related Hospitalizations, by Age Group and Specific Motor Vehicle - Traffic (MVT) External Causes, United States, 2002 - 2006

Table 13: Estimated Average Annual Numbers and Percentages of Traumatic Brain Injury-Related Hospitalizations, by Age Group and Expected Source of Payment, United States, 2002 - 2006 
Table 14: Average Annual Numbers, Rates, and Percentages of Traumatic Brain Injury-Related Deaths, by Age Group and Sex,

United States, $2002-2006$

Table 15: Average Annual Numbers, Rates, and Percentages of Traumatic Brain Injury-Related Deaths, by Age Group and Race, United States, $2002-2006$

Table 16: Average Annual Numbers, Rates, and Percentages of Traumatic Brain Injury-Related Deaths, by Age Group and External Cause, United States, $2002-2006$

Table 17: Average Annual Numbers, Rates, and Percentages of Traumatic Brain Injury-Related Deaths, by Age Group and Specific Motor Vehicle - Traffic (MVT) External Causes, United States, $2002-2006$

Figure 8: Annual Estimates of All Traumatic Brain Injury-Related Emergency Department Visits, Hospitalizations, and Deaths, United States, $2002-2006$

Figure 9: Annual Rate Estimates of Fall-Related Traumatic Brain Injury-Related Emergency Department Visits, Hospitalizations, and Deaths Among Children Aged 0 - 14, United States, 2002 - 2006

Figure 10: Annual Rate Estimates of Fall-Related Traumatic Brain Injury-Related Emergency Department Visits, Hospitalizations, and Deaths Among Adults Aged 65 and Older, United States, $2002-2006$

Appendix B: Methods and Data Sources

Table 18: ICD-9-CM Codes for Traumatic Brain Injury-Related Emergency Department Visits and Hospitalizations (2002 - 2006) 53

Table 19: ICD-10 Codes for Traumatic Brain Injury-Related Deaths (2002 - 2006)

Table 20: External Cause of Injury Categorization for ICD-9-CM Codes

(Emergency Department Visits and Hospitalizations, 2002 - 2006) and ICD-10 (Deaths, 2002 - 2006)

Table 21: Estimated Average Annual 2002 - 2006 Population by Age Group, Sex, and Race;

Weights for 2000 Standard Population by Age Group

Figure 11: Sources of Potential Case Duplication When Combining Traumatic Brain Injury-Related 


\section{REFERENCES}

1. Traumatic Brain Injury in the United States: Emergency Department Visits, Hospitalizations and Deaths. Atlanta (GA): Centers for Disease Control and Prevention, National Center for Injury Prevention and Control; 2004.

2. McCaig LF, McLemore T. Plan and Operation of the National Hospital Ambulatory Medical Care Survey. Centers for Disease Control and Prevention, National Center for Health Statistics. Vital Health Stat 1994;1(34).

3. International Classification of Diseases: 9th Revision, Clinical Modification, 3rd ed. (ICD-9-CM). Washington (DC): Department of Health and Human Services (US); 1989.

4. Arias E, Anderson RN, Hsiang-Ching K, Murphy SL, Kochanek KD. Deaths: Final Data for 2001. Centers for Disease Control and Prevention, National Center for Health Statistics. Natl Vital Stat Rep 2003;52(3).

5. International Classification of Diseases, 10th Revision (ICD-10). Geneva (Switzerland): World Health Organization; 2001.

6. Marr A, Coronado V, editors. Central Nervous System Injury Surveillance Data Submission Standards-2002. Atlanta (GA): Centers for Disease Control and Prevention, National Center for Injury Prevention and Control; 2004.
7. Thurman DJ, Sniezek JE, Johnson D, Greenspan A, Smith S. Guidelines for Surveillance of Central Nervous System Injury. Atlanta (GA): Centers for Disease Control and Prevention, National Center for Injury Prevention and Control; 1995.

8. Coronado VG, Thomas KE, Sattin RW, Johnson RL. The CDC Traumatic Brain Injury Surveillance System: Characteristics of Persons Aged 65 Years and Older Hospitalized with a TBI. J Head Trauma Rehab. 20(3): 215-228, 2005.

9. Langlois J, Kegler S, Butler J, Gotsch K, Johnson R, Reichard A, et al. Traumatic Brain Injury-Related Hospital Discharges: Results from a 14-State Surveillance System, 1997. MMWR Surveill Summ 2003;52 (No. SS-4):1-20.

10. Recommended Framework of E-code Groupings for Presenting Injury Mortality and Morbidity Data. Atlanta (GA): Centers for Disease Control and Prevention, National Center for Injury Prevention and Control; 2001. Available at: http://www.cdc.gov/injury/wisqars/ecode_matrix.html

11. U.S. Census Populations with Bridged Race Categories. Hyattsville (MD): Centers for Disease Control and Prevention, National Center for Health Statistics; 2003. Available at: http://www.cdc.gov/nchs/nvss/bridged_race.htm 
12. Miniño AM, Anderson RN, Fingerhut LA, Boudreault MA, Warner M. Deaths: Injuries - 2002. Centers for Disease Control and Prevention, National Center for Health Statistics; Natl Vital Stat Rep 2006; 54(10).

13. SAS Institute Inc. SAS/STAT User's Guide, Version 9.2. Cary (NC): SAS Institute Inc.; 2008.

14. Schootman M, Fuortes LJ. Ambulatory Care for Traumatic Brain Injuries in the U.S., 1995-1997. Brain Inj 2000; 14:373-81.

15. Sosin DM, Sniezek JE, Thurman DJ. Incidence of Mild and Moderate Brain Injury in the United States, 1991. Brain Inj 1996;10:47-54.

16. Adekoya N, Thurman D, White D, Webb K. Surveillance for Traumatic Brain Injury Deaths-United States, 1989-1998. MMWR Surveill Summ 2002;51(No. SS-10):1-16.

17. Sosin DM, Sniezek JE, Waxweiler RJ. Trends in Death Associated with Traumatic Brain Injury, 1979-1992. Success and Failure. JAMA 1995;273(22):1778-80.

18. Winn DW, Agran PF, Anderson CL. Sensitivity of Hospitals' E-coded Data in Identifying Causes of Children's ViolenceRelated Injuries. Public Health Rep 1995;110:277-81.
19. U.S. Advisory Board on Child Abuse and Neglect. A Nation's Shame: Fatal Child Abuse and Neglect in the United States. Report No. 5. Washington (DC): Department of Health and Human Services (US), Administration for Children and Families; 1995.

20. The National Elder Maltreatment Incidence StudyFinal Report. Washington (DC): Department of Health and Human Services (US), Administration for Children, Administration on Aging; 1998.

21. MacKenzie EJ, Steinwachs DM, Shankar BS. Classifying Trauma Severity Based on Hospital Discharge Diagnoses: Validation of an ICD-9-CM to AIS-85 Conversion Table. Med Care 1989;27:412-22.

22. Pickelsimer EE, Selassie AW, GU JK, Langlois JA. A PopulationBased Outcomes Study of Persons Hospitalized with Traumatic Brain Injury: Operations of the South Carolina Traumatic Brain Injury Follow-Up Registry. J Head Trauma Rehab 2006;21(6):491-504. 
U.S. DEPARTMENT Of HEALth AND HUMAN SERVices CENTERS FOR DISEASE CONTROL AND PREVENTION

\&

www.cdc.gov/TraumaticBrainInjury 